\title{
Non-isotropic self-assembly of nanoparticles: from compact packing to functional aggregates
}

\author{
Xavier Bouju* \\ CEMES-CNRS (UPR CNRS 8011), Bt F picoLab 29 Rue J. Marvig, BP 9434731055 Toulouse Cedex 4, France \\ Étienne Duguet* \\ CNRS, Univ. Bordeaux, ICMCB, UPR 9048, 33600 Pessac, France \\ Fabienne Gauffre* \\ Institut des sciences chimiques de Rennes (ISCR), UMR CNRS 6226, \\ 263 avenue du Général Leclerc, CS 74205, F-35000 Rennes, France \\ Claude Henry* \\ Centre interdisciplinaire de nanoscience de Marseille (CINAM), UMR CNRS 7325, \\ Aix-Marseille Université, Campus de Luminy, Case 913, F-13000 Marseille, France \\ Myrtil Kahn* \\ Laboratoire de chimie de coordination (LCC), UPR CNRS 8241, \\ 205 route de Narbonne, F-31000 Toulouse, France \\ Patrice Mélinon* \\ Université de Lyon, Université Claude Bernard Lyon 1, UMR CNRS 5306, \\ Institut Lumière Matière, F-69622, Villeurbanne, France ${ }^{\dagger}$ \\ Serge Ravaine \\ CNRS, Univ. Bordeaux, CRPP, UPR 8641, 33600 Pessac, France
}

(Dated: August 30, 2017)

\begin{abstract}
Quantum strongly correlated systems which exhibit interesting features in condensed matter physics often need unachievable either temperature or pressure range in classical materials. One solution is to introduce a scaling factor namely the lattice parameter. Synthetic heterostructures named superlattices or supracrystals were synthesized, including individual and collective use of semiconductors, metals and insulators for the exploitation of their unique properties. However, most of them are currently limited to dense packing. Unfortunately, some desired properties need to adjust the colloidal atoms neighbouring number. This review sums up the current state of the research in non-dense packing, discusses the benefits, outlines possible scenarios and methodology, describes examples reported in the literature, briefly discusses the challenges, and offers preliminary conclusions. Penetrating such new and intriguing research field demands a multidisciplinary approach accounting for the coupling of statistic physics, solid state and quantum physics, chemistry, computational science and mathematics. Standards interactions between colloidal atoms and emerging field such as Casimir forces use are reported. In particular, the paper focuses on the novelty of patchy colloidal atoms to meet this challenge.
\end{abstract}

\section{CONTENTS}

I. Introduction

3

II. glossary

4

III. Interactions between particles

A. Dipole-Dipole Interaction

5

B. Dispersive van der Waals Interactions

C. Electrostatic Interactions

\footnotetext{
* Observatoire des micro et nanotechnologies (OMNT), Minatec 17 rue des Martyrs, F-38000 Grenoble, France

† patrice.melinon@univ-lyon1.fr
}

D. Magnetic Forces

E. Steric Interaction $\quad 6$

F. Depletion Interactions 6

G. The effect of water for hydrophobic NPs 6

H. Molecular Surface Forces 6

I. Casimir Forces: a survey $\quad 7$

1. Quantum phase transition: Casimir-Polder and Casimir-Lifshitz Forces

2. Classical phase transition:

Casimir-deGennes-Fisher forces

3. CDF potential in binary mixture

J. Ligands

1. Ligand-NP and ligand-ligand interaction (6) (1) . . 
2. Modelling patch: anisotropic interaction

3. DNA

K. Effect of the particle size and shape

IV. Driving Forces in self-assembly of particles $\quad 10$

A. Particles without interactions 10

B. Particles with interaction: crude model 10

C. Particles with interaction in binary mixture

D. External driving force

V. NPs synthesis
A. Strategy
B. Nucleation of colloidal crystals: theory
C. Mechanism of growth in the framework of the La Mer model

VI. Supracrystal based on spherical (isotropic) particles

A. Size dispersity

B. Topology

C. Crystallization

D. Particles with interaction

VII. Supracrystal based from non-spherical particles: theory and computer simulation of particle self-assembly

A. Optoelectronics: photonic crystals

B. Metamaterials 26

C. Plasmonics 27

D. Application in heterogeneous catalysis 27

E. Magnetism 28

F. Energy storage

G. Mechanical properties of TPMS supracrystal

1. outlook

2. Mechanical properties 30

$\mathrm{H}$. The future of additive manufacturing $\quad 30$

XI. Conclusion/Perspectives 31

References

A. Theory of self-assembly 16

B. Computer simulation 17

C. Comparison between theoretical models and experiment: case of polyhedron particles 17

1. Tetrahedra 17

2. Octahedra 18

3. Cubes 18

4. Cuboctahedra 19

5. Truncated cubes 19

6. Truncated octahedra 19

7. Rhombic dodecahedron 19

VIII. Anisotropy: bimodal distribution 19

A. Isotropic particles 19

B. Mixture of isotropic/anisotropic particles 20

IX. Supracrystal based on anisotropic

functionalized particles with interactions $\quad 21$

A. Numerical self-assembly of patchy hard spheres

B. Synthesis and self-assembly of particles bearing two and more patches 23

C. DNA 24

1. NPs directionnaly functionalized with DNA 24

2. Origamis 24

3. DNA transplant onto NPs with patchs 25

X. (expected) properties of anisotropic self-assemblies of Nps and possible applications 


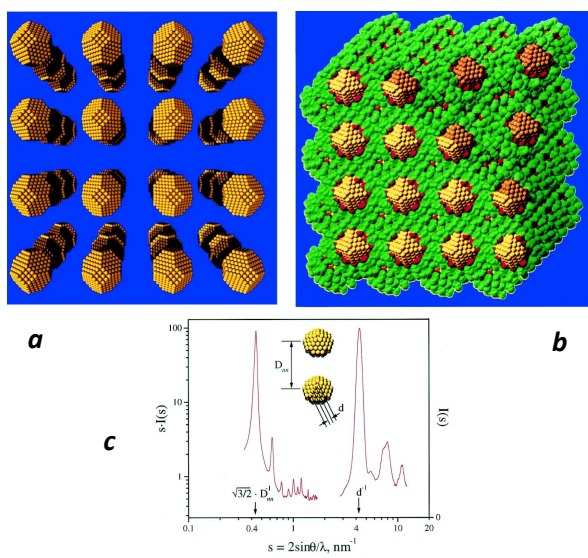

FIG. 1. Giant aurothiol cluster (c-Au:SR) compound, drawn to scale forming a cubic supracrystal (a) (the central columns of nanocrystals from a body-centered-cubic type structure have been removed for clarity of viewing) [7] according to the synthesis method proposed by Brust and Schiffrin [8]. The compound's core, at center, is represented in idealized form consisting of $144 \mathrm{Au}$ atoms (golden spheres). 62 n-butylthio groups adsorbed (red) sulfur atoms; (green) methyl(ene) groups are displayed in the right side (b). The diffraction pattern (c) reveals the superlattice parameter (after the original figure in [7]).

\section{INTRODUCTION}

Quantum strongly correlated systems which exhibit interesting features in condensed matter physics such as phase transitions in high temperature superconductivity, spin ordering in magnetism, superfluidity, Bose-Einstein condensation, etc, often need unachievable either temperature or pressure range [1] in classical materials. One solution is to introduce a scaling factor namely the lattice parameter. For that purpose, atomic physics developed cold atoms in optical lattices used to simulate quantum phenomena and models in condensed matter physics [2]. This interest in these superlattices (cold atoms organized as supracrystals) emanates from the growing advancement in techniques to prepare, manipulate and detect strongly correlated states in them. However, this wonderful toolbox is limited to the laboratory so far. Applications in the "daily life" necessarily requires the synthesis of materials having large lattice parameters in standard conditions of pressure and temperature. This explains why assembling atoms into clusters which can then be used as building blocks to form supracrystals (figure 1) has undergone tremendous development over the past years [3-6].

For a long time, the isotropic character of the particles associated to the common interacting forces [9] limited supracrystal to the most compact architectures , face-centered cubic (fcc) or hexagonal-close packed (hcp), as described in the so-called Derjaguin-Laudau-VerweyOverbeck (DLVO) theory. Novel strategies based from patch and specific oriented ligands including DNA open now a large field of architectures more or less dense templating the Bravais lattices. Nanocrystal size, interparticle distance, and coating agent play key roles in supracrystal properties $[10,11]$. Some parameters cannot be easily tuned in a wide range. The transition between different symmetries and bond lengths within an atomic crystal requires a phase transition at high temperature $T$ or pressure $P$ where the stability is not achieved in normal conditions. This is not the case in supracrystals where lattice symmetry and parameter can be adjusted in a wide range without recourse to external $P$ and $T$. However, this is true as long as a supracrystal templates a Bravais lattice $[12,13]$. It is generally admitted that atoms are assimilated to spheres. The opened question is what happens when spheres are replaced by complex polyhedra with specific shape symmetries. They form superlattices where a particular class called metamaterials are periodic at a much larger scale than the building unit. At a first glance, superlattices are simply crystals with unusual (large) periodic lengths. These materials have strong effects over periodic fields having a wavelength close to the crystal period. When the periodicity matches with the excitation wavelength, superlattices form photonic band gaps or phonon band gaps or other equivalent systems. The key point is the diffraction related to resonance effect which creates band gap inside the lattice. This is more complicated since the spheres are replaced by polyhedra that introduce new degrees of freedom. First, the polyhedra have their own lattice space group symmetry and a shape with a specific point group symmetry. The open-ended question is how matches the polyhedra point group symmetry and the superlattice one: are they commensurable or incommensurable? The first issue comes from the well-known fullerite $\mathrm{C}_{60}$. Solid $\mathrm{C}_{60}$ is a molecular crystal in which $\mathrm{C}_{60}$ molecules occupy the lattice sites of a fcc $(F m \overline{3} m)$ structure where van der Waals interactions are the dominant intermolecular forces. Then, near the temperature $T_{c} \sim 250-260 \mathrm{~K}$, the $\mathrm{C}_{60}$ crystal is known to undergo a first-order phase transition from fcc to simple cubic $(P \bar{a} 3)$ associated with changes in the molecular rotations [14]. Above $T_{c}, \mathrm{C}_{60}$ molecules rotate almost freely templating a "super spherical atom". Below $T_{c}$, the molecular rotations are partially locked with the fivefold symmetry axes having specific orientations (see Fig. 2a). Since fivefold group $\left(I_{h}\right)$ is incompatible with $(F m \overline{3} m)$ space group [15], the $\mathrm{C}_{60}$ solid at low temperature adopts a common point subgroup $T_{h}$ ( $P \bar{a} 3$ space group) leading unusual features as observed in the transport properties [16, 17]. Another issue is the coupling between clusters since the links between them can be tuned from few atomic lengths to microns. About optical properties, the most emergent effect is the broadband spectral tunability of the collective plasmonic response ranging from strongly coupled clusters to isolated ones (Fig. 2b) [18, 19].

The last issue is the symmetry of the superlattice including the first neighboring number $n_{c}$. For instance, transport properties are related to metal insulator tran- 


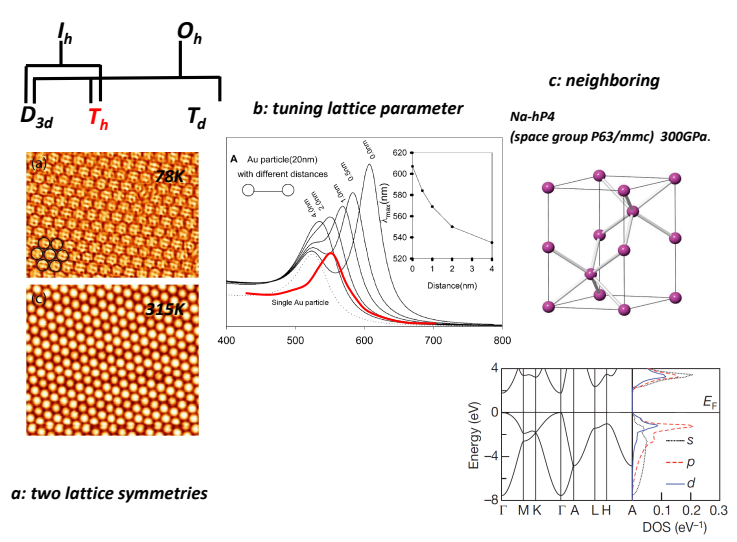

FIG. 2. Three effects in supracrystal architectures (a) cluster point group symmetry versus supracrystal point group symmetry: the case of $\mathrm{C}_{60}$ fullerite observed by scanning tunneling microscopy (STM) below and above the critical temperature [20]. After freezing the supracrystal lowers its symmetry according to the subgroup table. (b) Plasmon shift calculated as a function of the distance between two NPs after the reference [18]. (c) Metal insulator transition in sodium bulk. Sodium is insulating at very high pressure with lowering its coordination number from 8 in normal conditions to 6 . The insulator character is partially due to the s-p-d hybridization $[21]$.

sition. According to the Mott-Hubbard common picture [22], the metal like is achieved for $W / 2>U$ where $W$ is the bandwidth and $U$ the correlation energy. Since the bandwidth is proportional to the square root of the neighboring number (here the cluster neighboring number), transport properties can be tuned by $n_{c}$. This is observed in common lattices but in severe conditions needing generally high pressure/temperature conditions such as in transparent dense sodium at $300 \mathrm{GPa}$ [21] (Fig. 2c).

The original idea behind the present review is to give developments and strategies for synthesis of directional building blocks (faceted or limited valence colloidal particles) and the control of the toolbox of the assembly forces (attractive or repulsive, short or long range, selfcorrecting / reversible or not ...). In a first part, we deal with a brief overview of the most relevant strategies described in the literature to date affording isotropic and therefore close-packed assemblies: it will allow to precisely define the many driving forces available to assemble the micro and nano building blocks. A special attention will also be devoted to establish the typical vocabulary (glossary) of this topic (e.g. concept of colloidal atom or colloidal crystals, ). The heart of the paper focuses on chemical and physical engineering developed for the synthesis of the elementary anisotropic building-blocks and (very) first non-compact self-assemblies. Self-assembly mechanisms and the contribution of numerical modelling are discussed. The main issue is the selective functionalization of their oriented surface (using organic ligands, DNA fragments, micro (and nano) patchy particles etc.).
The last part is dedicated to some current examples and new perspectives offered by supracrystals in many fields, crystallography, plasmonic, magnetism, chemistry, catalysis, etc.

\section{GLOSSARY}

A special attention will be devoted to establish the typical vocabulary used in the text.

Bravais lattice: in common crystals, the set of all possible ways a lattice can be periodic if composed of identical spheres placed at the lattice point form the 14 Bravais lattices.

Colloid: a colloid is constituted of a dispersed phase, i.e. the nanoparticles (NPs), and a continuous phase, i.e. the solvent, the mixture of which should be stable over time.

Colloidal atom: this term refers to a colloidal object (particle) which is used as a building-block to form a supracrystal (colloidal crystals), by analogy with atoms forming crystals.

Colloidal crystals: colloidal crystals consist in an ordered array of NPs (e.g. gem opal). Superlattices, superlattice, and supracrystals refer to the same concept.

Flory-Huggins parameter: this parameter $(\zeta)$ is introduced in the theory of polymer solutions to take into account the energy of mixing monomer units and solvent molecules. Good solvents have a low $\zeta$ and poor solvent a high $\zeta$.

Kagome lattice: a lattice formed by the vertices and edges of interlaced triangles (two interlaced triangles form a David star).

Ligands: a ligand is a species (i.e. molecule with functional group) that forms at least one bound with an atom of the nanoparticle surface. As in coordination chemistry, the nature of the bonding can range from covalent to ionic.

Monodisperse: dispersity is a measure of the heterogeneity of sizes of molecules or particles in a mixture. A collection of objects is called uniform if the objects have the same size, shape, or mass.

Nanocrystals: nanocrystals consists in crystalline nanoparticles in contrast to amorphous ones.

Self-assemblies: self-assemblies result from the organization of a disordered system that forms an organized structure through specific, local interactions among the species constitutive of the system themselves [23, 24].

Order parameter: an order parameter is a physical length (generally normalized to remain in the range $[0-1])$ which evolves during a phase transition. It can be density in a liquid/gas transition or the average magnetization for magnetic transitions. Generally the order parameter will be 0 in the less organized phase and 1 in the organized or condensed phase.

Percolation: during a first order transition, islets of the new phase nucleate into the initial one and grow. 
Percolation correspond to the situation when all domains of the growing phase are interconnected.

Point group symmetry: it is the group of symmetries that leaves a crystal (or a molecule) invariant. They are used to make categories of crystals relative to their geometry.

Zeta-potential: colloids in solution are surrounded with an ionic double layer. In their motion, they drag with them a part of this ionic layer, which is immobilized at the surface. The zeta-potential is the electric potential difference between the bulk and this slippery interface.

\section{INTERACTIONS BETWEEN PARTICLES}

The key fonction when one deals with assembly is the interaction between building blocks. Regarding the size of these building blocks, various types of forces are at the origin of the assembled structures. For molecular selfassembly for instance, the relative strength of the interactions generates motifs that can be tuned according to the molecular specificity of the units [25]. At a larger scale, some interactions vanish (hydrogen bonding, covalent interaction, substrate-mediated interaction...) whereas others are averaged (electrostatic and magnetic interactions by example) or get a new form (van der Waals and Casimir forces...) [26-28]. In this section, we give a short overview of the forces between particles with sizes ranging from the nanoscale to the microscale.

\section{A. Dipole-Dipole Interaction}

A permanent dipole may exist within the material due to an inhomogeneous distribution of electrons. For instance, elongated gold NPs with (111) and (100) facets placed in a solution of citrate and mercaptoethanol molecules will exhibit a permanent dipole due the preferential adsorption of citrate on one type of facets. In consequence, large dipolar moments will affect the interactions between nanoparticles (NPs) through dipolar coupling. Equation 1 corresponds to the potential energy between two dipoles possessing a moment $\mu$ and distant of $r$ :

$$
V_{\text {dipole }}=-\frac{\mu^{2}}{4 \pi \epsilon_{0} \epsilon r^{2}}\left(2 \cos \theta_{1} \cos \theta_{2}-\sin \theta_{1} \sin \theta_{2} \cos \phi\right)
$$

where the orientation of the dipoles $i$ with respect to a main axis is described by $\theta_{i}$ and $\phi, \epsilon$ and $\epsilon_{0}$ are the permittivity of the NP material and of the vacuum, respectively [29]. Depending on the relative orientation of the dipole inside the nanoparticle, the configuration in which the two dipoles are inline or antiparallel is favored. Dipole-dipole interactions scale like $r^{-3}$ and could explain the phase diagram observed in some nanocrystal superlattices [30].

\section{B. Dispersive van der Waals Interactions}

The van der Waals forces (vdW) gather three contributions [29]: the Keesom contribution between two fixed dipoles (cf. Sect. III A), the Debye contribution between a rotating dipole and a nonpolar entity, and the London contribution between two instantaneous dipoles. This last term is a dispersive interaction and stands generally for $\mathrm{vdW}$ interaction. It originates from electromagnetic fluctuations due to the relative movements of positive and negative charges inside the atoms. They mainly generate an attractive force and play an important role in the selfassembly of particles. In some very special cases repulsive $\mathrm{vdW}$ forces have been predicted and experimentally measured [31]. However, the exact treatment of vdW forces is very difficult and an approximate treatment based on the Hamaker theory is generally used [32]. It consists in a summation of pairwise interactions between atoms belonging to two macroscopic bodies like two NPs under the form of an integration over the volume of the interacting particules. An accurate treatment of $\mathrm{vdW}$ forces is the continuum Dzyaloshinskii-Lifshitz-Pitaevskii (DLP) theory [33]. However application of the DLP method is limited for very small or anisotropic particles for which the dielectric response deviates from bulk values. In this case another method can be used: the Coupled-Dipole Method [34-36] which is a more relevant atomistic treatment.

Note that the van der Waals potential depends on the shapes of the NPs. For two isotropic NPs with a radius $R$ and a distance $r$ between the two NPs centers, the associated non-retarded interaction energy is:

$$
V_{v d W}=-\frac{H}{3}\left[\frac{R^{2}}{r^{2}-4 R^{2}}+\frac{R^{2}}{r^{2}}+\frac{1}{2} \ln \left(1-\frac{4 R^{2}}{r^{2}}\right)\right],
$$

where $H$ is the Hamaker constant of the NPs material. One recovers the limit case, when $R \ll r$ then $V_{\mathrm{vdW}}=$ $-(16 H / 9)\left(R^{6} / r^{6}\right)$.

\section{Electrostatic Interactions}

Electrostatic interactions involved dissociated charges. Charges dissociate easily in polar media due to the high dielectric constant ( $\epsilon \sim 80$ for water) while they do not dissociate easily in nonpolar solvents. This does not imply that charges are absent and that these effects should be neglected in nonpolar solvents. They can be either attractive or repulsive. The amplitude and the length scale of the interaction can be controlled by choosing the appropriate solvent with surrounding counterions. For example, the scarcity of charges induces a lack of screening of electrostatic interaction; charges will have effect over large distances, a small double-layer capacitance will exist and significant surface potentials will be generated. The electrostatic forces $V_{e l}$ between particles are generally modelled by the DLVO approximation [37-39]. 


$$
V_{e l}=\frac{\epsilon_{0} \epsilon R^{2} \xi^{2}}{R \star} \exp [-\kappa(r-2 R)]
$$

where $R$ is the sphere radius, $\kappa$ is the Debye length, $\xi$ is the $\xi$-potential. One important question is from where the charges may come from? In colloidal nanocrystal system, ligands that are bound to the particles surface should compensate for the charges carried by the surface ions. Obviously, defects in this surface coverage can induce charges. Interestingly, the structural diversity of binary NPs superlattices composed of two NPs with resulting charges of opposite sign is reported to be induced by electrostatic interactions [40]. This model is inaccurate for the small interparticle distances [27]. By adjusting electrostatic forces like in changing the screening length it has been possible to self-assemble nanoparticles in non-compact diamond structure [41] or to fabricate a binary cocrystal by using anionic and cationic species [42].

\section{Magnetic Forces}

When particles present a permanent magnetic moment, directional interactions appear which can be attractive or repulsive. They are modeled by a dipoledipole interaction. If the particles are superparamagnetic the interaction can be triggered by an external magnetic field and the magnetic interaction between two particles separated by the distance $r$ and with a magnetic moment $m_{m}$ is:

$$
V_{\mathrm{magn}}=-\frac{\mu_{0} m_{m}^{2}}{2 \pi r^{3}}\left(1-\frac{3}{2} \sin ^{2} \theta\right)
$$

where $\theta$ is the angle between the external field and the line joining the sphere centres [43, 44]. Interestingly, a dual application of electric and magnetic fields on superparamagnetic particles allows the formation of birectional chains, colloidal networks and crystals by modulating the electric field and by keeping uniform the magnetic field [45]. On the other hand, magnetic cubic nanoparticles can form 1D, 2D, 3D structures such as chains, ribbons, and large cuboids that are self-assembled with a combinaison of dipole-dipole magnetic interaction and vdW interaction [46].

\section{E. Steric Interaction}

A repulsive force between two NPs stabilized by ligands exist when these NPs approach at a distance smaller than twice the ligand-brush length due to ligand compression. This will impact the free energy. For planar surfaces:
$\Delta G=2 \mu k_{\mathrm{B}} T V(r)+2\left(\frac{2 \pi}{9}\right)^{3 / 2} \mu^{2} k_{\mathrm{B}} T\left(\alpha^{2}-1\right)\left\langle r \star^{2}\right\rangle M(r)$,

where $\mu$ is the density of ligand, $\alpha$ is an expansion factor related to the Flory-Huggins parameter and depending on the solvent, $\left\langle r \star^{2}\right\rangle$ is the mean length of the ligand in solution, and $V(r)$ and $M(r)$ are functions depending on the distance between the NPs only. Note that in the case of spherical NPs, $\Delta G$ is more complicated as the curvature of the NPs has to be taken into account. However, Eq. 5 remains valid when the ligand-brush length is much smaller than the NPs radius [47]. Other theoretical approaches have been developed to take into account these ligands induced interactions [48-51].

\section{F. Depletion Interactions}

The origin of this force is purely entropic [52]. An attraction between the colloids that is of osmotic origin is induced by the depletion of the ligands between the NPs. Such effect is observed when two NPs become close enough in a system containing NPs and smaller objects (e.g. organic molecules like ligands, polymers). The potential corresponds to the product of the osmotic pressure induced by the depletant and the overlap volume. Such interactions have been used to induce the self-assembly of NPs [53-56].

\section{G. The effect of water for hydrophobic NPs}

If the presence of water in organic solvents is often neglected, it may have several different effects on the interactions between NPs. For examples, water can form reverse micelles in nonpolar solvents, adsorb at the surface of NPs, or template the formation of hybridosomes [57-60].

\section{H. Molecular Surface Forces}

Many other forces occur between the surfaces of the particles like covalent bonds, hydrogen bonds, dipolar interactions. Hydrogen bonds are particularly important between complementary DNA base pairs [61]. These interactions can be turned on or off by changing the temperature. By placing the DNA bases at some specific positions on the particles, directional interactions are obtained which have been recently used to make nanoparticle superlattices with the diamond structure [62]. Another important type of molecular surface force is due to the presence of ligands surrounding the particles. They are of different kinds. The forces between ligands when two particles become close is repulsive (steric repulsion) and the entropy of the solvent molecules increases by the 
partial breaking of solvent shell leading to an attractive interaction.

\section{Casimir Forces: a survey}

Confined fluctuating fields induce forces upon enclosing surfaces. This situation can be extended to classical statistical physics where the order parameter plays the role of the fluctuating field. Close to a Landau secondorder phase transition, where the correlation length diverges, these forces take universal scaling forms characterized by the symmetry of the system, the dimensionality and the boundary conditions. Among them, CasimirLifshitz and Casimir-deGennes-Fisher forces play a role in the colloidal chemistry.

\section{Quantum phase transition: Casimir-Polder and Casimir-Lifshitz Forces}

In quantum field theory, the Casimir-Polder [63] (CS) forces are physical forces arising from a quantized field. Their origins lie into the fluctuation of the electromagnetic field in vacuum and are directly related to Heisenberg principle. Contrary to van der Waals forces where a fluctuating dipole induces a fluctuating electromagnetic dipole field, which in turn induces a fluctuating dipole on a nearby particle, CS forces concern large distances between particles with retardation effects. In the seminal work of Casimir and Polder [63, 64], the calculation was done in vacuum between two parallel plates with an infinite dielectric constant (perfect conductor). In fact, due to the skin depth and the plasmon frequency above which the conductivity goes to zero, the CS forces were experimentaly evidenced [65][66] for a critical distance $d_{c}>1 \mathrm{~nm}:$

$$
d_{c}>\left(\frac{m e}{\alpha 4 \pi \hbar n}\right)^{1 / 2}
$$

where $n$ is the density of free electrons in the metal, $\alpha$ the fine structure constant. However, Casimir's analysis of idealized metal plates was generalized to arbitrary dielectric (for example a solution) and realistic metal plates (or spheres in a colloidal solution) by Lifshitz [67]. In a colloidal solution, Casimir-Lifchitz forces (CL) are effective for a distance to $1 \mathrm{~nm}$ up to few tenths of nanometers $[68,69]$. There are few instances wherein the CasimirLifshitz effect can give rise to repulsive forces between colloids in the solution [70].

\section{Classical phase transition: Casimir-deGennes-Fisher forces}

A more generic route to temperature control of colloidal phase behavior is to suspend colloids in a nearcritical binary liquid. Figure 3 displays the formation

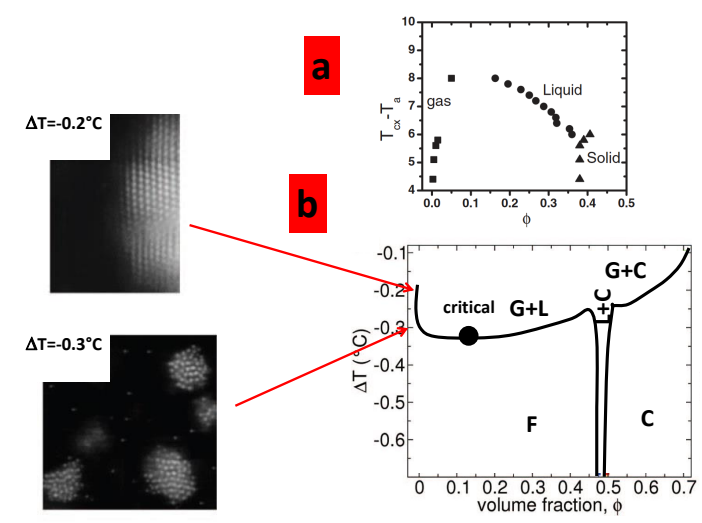

FIG. 3. (a) Phase diagram of polystyrene spheres suspended in a binary liquid mixture of 3-methylpyridine and water, $T_{c x}$ is the critical temperature, $\phi$ the volume fraction of spheres (after the original figure of [71]. (b) Colloidal phase diagram induced by the Casimir-deGennes-Fisher forces for poly-nisopropyl acrylamide (PNIPAM) particles with a diameter of $500 \mathrm{~nm}$ suspended in a near-critical quasi-binary solvent composed of 3-methyl pyridine (3MP), water, and heavy water system and snapshots of confocal microscope observations for two temperatures showing a lattice formation, G gas, L liquid, F fluid, S solid. Adapted from the original figure in [72], this phase diagram can be compared to the one in Fig. 14.

of solid and liquid colloidal phases in equilibrium with a dilute gas phase with only the temperature as a control parameter [71, 72].

The confinement of the critical fluctuations of the order parameter close to the critical point gives rise to an effective force between the colloids which exhibits striking similarities with the Casimir force in quantum field theory [73] (see Fig. 8c), and is sometimes called critical Casimir force [74]. Casimir-deGennes-Fisher force (CDF) induced interactions are generally thought to drive particle aggregation and colloidal self-assembly processes in a tunable, reversible, and in-situ fashion due to their strong dependence on temperature and on solvent composition [75-79]. The advantage of the CDF effect is its universality: as other critical phenomena, the scaling functions depend only on the symmetries of the system and are independent of colloidal properties.

\section{CDF potential in binary mixture}

We now back to the repulsive term in DLVO theory for binary liquid mixtures. For two particles of diameter $2 R$ at close center-center interparticle separation $r$, the repulsive electrostatic potential has the general form [72]:

$$
U_{\text {rep }}(r)=A_{\text {rep }} \exp \left(-r / \lambda_{D}\right)
$$

where $A_{\text {rep }}$ is the amplitude and $\lambda_{D}$ the Debye screening length. The Casimir-like attraction between two spheres 


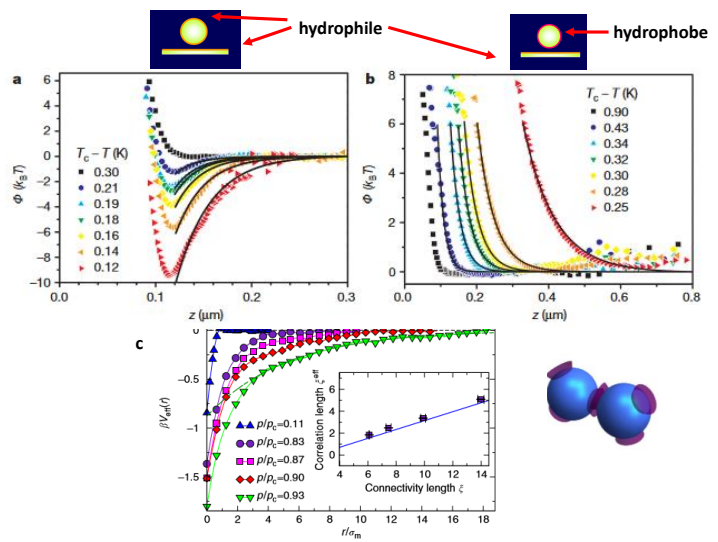

FIG. 4. Top: CDF force observed between a coated colloidal sphere (polystyrene) and a planar surface immersed in a binary liquid mixture of water and 2,6-lutidine near the critical point showing the amplitude and the sign of the force [75]. The amplitude is tuned by the shit of the temperature with respect on the critical one and a particle in a critical water/lutidine mixture. A solvent composed by cosolutes (water/lutidine) of different size could introduce a structure in the effective potential controlled by the different cosolute length scales. Cosolutes are constituted by a chemical sol that is, a sol composed by particles linked into clusters through irreversible bonds. Effective forces between polystyrene particles are controlled by the clustering properties of the solvent. (a) Symmetric boundary conditions with hydrophilic particle and $\mathrm{NaOH}$-treated hydrophilic wall corresponding to attractive force. (b) Asymmetric boundary conditions where polystyrene particle preferring lutidine (hydrophobe) and $\mathrm{NaOH}$-treated hydrophilic wall. (c) (bottom) Monte Carlo simulation of attractive force between hard sphere (sol packing fraction 0.052) near the percolation threshold $p_{c}=0.682$ [77]. The value of " $p$ " is monitored by the temperature. The colloids with patch have a connnectivity of three. Adapted from Refs. [75] and [77].

writes [72]:

$$
U_{\mathrm{att}}(r)=\frac{A_{\mathrm{att}}}{\xi(T)} \exp [-r / \xi(T)]
$$

where $\xi(T)$ is the temperature dependent correlation length of the solvent [72],

$$
\xi(T)=\xi_{0}\left(1-T / T_{C}\right)^{-0.63} .
$$

$T_{C}$ is the critical temperature. CDF potential takes advantage that repulsive or attractive term can be modulated by the temperature as long $U_{\text {att }}(r)$ is temperature dependent as depicted in Fig. 4.

The keypoint of the CDF force originates from the confinement of the solvent critical fluctuations between the surfaces of distinct colloids and belongs to the class of the so-called critical parameter which exhibits scaling and universality [73]. Then, the interaction between colloidal hard sphere particles immersed in a chemical solution becomes attractive and long-ranged on approaching the

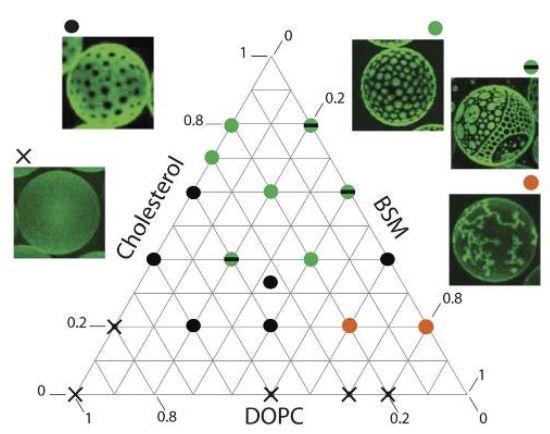

FIG. 5. Immiscibility diagram for ternary mixtures of 1,2dioleoyl-sn-glycero-3-phosphocholine (DOPC) - brain sphingomyelin (BSM) - cholesterol stabilizing monodisperse emulsion droplets. The patterns are classified into different categories: bright spots on a dark background (green circles); dark spots on a bright background (dark circles); bright tree-like structures on a lighter back- ground (orange circles); mixtures of bright and dark spots (green circle with a black line); homogeneous surface (crosses). After the original figure in Ref. [83].

solution percolation transition. The analogy with CDF force is simply obtained by comparison between $T-T_{c}$ with $p-p_{c}$ ( $p_{c}$ being the percolation threshold) and $\xi$ the length of the infinite cluster nearby the transition (see Fig. 4).

\section{J. Ligands}

\section{Ligand-NP and ligand-ligand interaction}

Ligand is an ion or molecule attached to a metal atom at the cluster surface by coordinate bonding. Bonding may include several types of interactions such as electrostatic, hydrogen bonding, biological recognition interactions $[80,81]$. When the interaction depends on the type of crystal facet, we can create bond directionality which is the base of patch [82]. It is the case when phase separation occurs at the NPs surface due to miscible/immiscible ligand. Figure 5 illustrates the immiscibility phase diagram for ternary mixtures when immiscible lipids control the morphology of patchy emulsions [83].

\section{Modelling patch: anisotropic interaction}

Patchy colloidal atoms are particles decorated on their surface by a predefined number of attractive sticky spots [84-88]. Most of patchy colloidal atoms are obtained by chemical route. However, shell coating device can be obtained directly in free phase as depicted in Fig. 6 


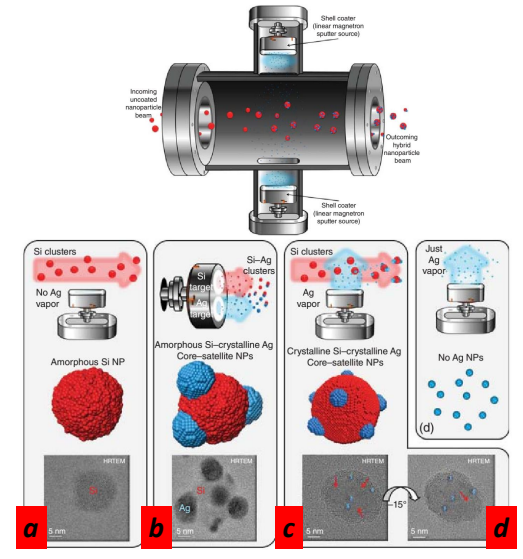

FIG. 6. Top: schematic representation of shell coating device where core nanoclusters are coated with shell material atoms. Here, core nanoclusters are produced in a magnetron sputtering source. (bottom) Schematic comparison between different configurations ((a)silicon clusters without silver atoms, (b) silicon and silver clusters, (c) silicon clusters with silver atoms, (d) silver atoms). Nano silicon clusters with patchs (silver clusters) can be produced in cases (b) and (c) after the original figures of [89].

[89]. The challenge is to introduce non-isotropic interactions between particles [90]. This makes it possible to achieve phase transition with arbitrary small packing fraction which can not be realized via the isotropic potentials mentioned above. Interactions between patchy particles can be looked as a covalent-like bonding. The most simple simulation of such potential writes [91, 92]:

$$
V_{\mathrm{eff}}(\mathrm{A}, \mathrm{B})=V^{\mathrm{HS}}(\mathrm{A}, \mathrm{B})+\sum_{i=1}^{f} \sum_{j=1}^{f} V_{\mathrm{SW}}\left(r_{\mathrm{AB}}^{i j}\right) .
$$

$V_{\text {eff }}(\mathrm{A}, \mathrm{B})$ is the interaction potential between two patched particles $\mathrm{A}$ and $\mathrm{B}, f$ the number of patches, $V^{\mathrm{HS}}(\mathrm{A}, \mathrm{B})$ the isotropic hard sphere model described above (DLVO, Casimir-like, Asakura-Oosawa ...). Two patchy particles interact through an attractive square well (SW) potential only if two patches are properly aligned. The interaction between patches is modelized by a square well potential $V_{\mathrm{SW}}$ of depth $U_{0}$ for $\delta \leq 0.12$ (see Eq. 12), $R$ being the radius of a (monodisperse) particle. Table I displays the gas-liquid coexistence regions for different packing and $f$ values. We clearly observed that the critical packing collapses when $f$ decreases [91]. Surprisingly, this value slowly decreases when the patches are randomly distributed [92]. Since the packing limit can be tuned by $f$, the existence of a region of densities which is not affected by phase separation is a characteristic of patchy interacting particles systems. This feature opens the way to non-dense packing superlattices [93, 94] such as diamond lattice.
TABLE I. Critical point parameters for different number of patches $f[91,92] .\langle f\rangle$ is a mean value, with $\delta=0.12$. The values can be compared to those in Tab. II.

\begin{tabular}{|l|l|l|l|l|l|l|l|l|}
\hline$\langle f\rangle$ & 2.43 & 2.49 & 2.56 & 2.64 & 2.72 & 3 & 4 & 5 \\
$\phi_{c}$ & 0.036 & 0.045 & 0.052 & 0.055 & 0.059 & 0.07 & 0.14 & 0.175 \\
\hline
\end{tabular}

\section{3. $D N A$}

Oligonucleotides, i.e. short single strands of DNA, have been recognized as ligands of choice for the assembly of NPs. Indeed, base pairing between complementary DNA strands yields an "intraparticle bond" with unequaled advantages [95, 96]. Thus, control of interparticle distance with subnanometer resolution is obtained, based on the number of base pairs in the DNA duplex. The tunable sequence-dependent enthalpy of interaction allows access to equilibrium structure, proof reading and error correction of self-assembly through thermal annealing procedure [95, 97]. In addition, sequence recognition also enables sequential binding [4,98]. When grafted into a densely packed shell, the DNA duplex arranges in a surface-normal orientation following precisely the shape of the nanoparticle, which is of particular interest for nonspherical nanoparticles. Post-synthesis addition of DNA intercalators into DNA duplexes increases the thermal stability of the DNA nanoparticle superlattices enabling further high-temperature modification of the assemblies [99]. In principle, DNA can be attached to many types of nanoparticles, including quantum dots, iron oxides and silica. However in most cases, DNA was attached to gold nanoparticles. Indeed, thiolated DNA can be easily anchored to the surface in a post-synthetic step, via the formation of S-Au bond [100]. In addition, gold nanoparticles can be produced with a variety of forms including rods, triangular prisms with edges, corner and facets that can be functionalized selectively [95].

\section{K. Effect of the particle size and shape}

The direct application to NPs of the self-assembly theory elaborated for microparticles (classical theory of colloidal interactions) is not straightforward. The classical approach of microparticles self-assembly uses the DLVO theory which makes several assumptions that are no longer verified for nanoparticles with sizes about $1-20$ $\mathrm{nm}$ [101]. First, the elementary interactions are no longer additive. Second, the size of the solvent molecules and solvated ions is not negligible compared to the size of the particles. Third, the media inside or outside the particles cannot be considered as uniform. For example, the ligand surrounding the particle has different propertiescompared to the particle core. Thus, it is not possible to consider a uniform dielectric constant instead we have to consider local atomic polarizabilities. Finally, the DLVO theory considers simple particle shapes, mainly spheres 
or less often cylinders while nanoparticles have a large palette of shapes, some of them being very complex. In particular, the presence of sharp apexes leads to singularities that affect the properties of the particles. In order to take into account above limitations, the different interactions have to be calculated simultaneously. This can be done by density functional theory (DFT) calculations or molecular dynamics (MD) simulations [101].

\section{DRIVING FORCES IN SELF-ASSEMBLY OF PARTICLES}

The interactions between particles play a central role in the assembly of particles. In the case of hard particles which has been studied for years there is no attraction between the particles only an infinite repulsion when particles come on contact. The free energy is minimized by the maximization of the entropy [102]. Entropic interactions (mainly depletion interactions) are the driving force for self-assembly of isotropic particles at large volume fraction $(\phi)$ giving compact structures (fcc, bcc, or hcp). For the hard spherical particles the fcc structure was conjectured by Kepler, four centuries ago [102] and proved by Hales only recently [103]. The situation is more complicated for anisotropic particles [104]. Directional entropic forces arise from the alignment of flat facets that maximizes entropy [105]. In the case of real particles other interactions are to be accounted. They are of van der Waals, electrostatic, magnetic or molecular types. A complete description is given in [27]. Here, we will give only the main characteristics of the interactions.

\section{A. Particles without interactions}

Hard spheres are widely used as model particles in the statistical mechanical theory of fluids and solids. They are defined simply as impenetrable spheres (radius R) that cannot overlap in space. The pairwise interaction potential is modelled as:

$$
V(r)=\left\{\begin{array}{lll}
0 & \text { if } & \mathrm{r}>\mathrm{R} \\
\infty & \text { if } & \mathrm{r}<\mathrm{R} .
\end{array}\right.
$$

Since there is no attractive part, the lowering of the free energy is achieved by maximising entropy.

\section{B. Particles with interaction: crude model}

The interaction bretween particles can be modelled by a square well potential:

$$
U(r)=\left\{\begin{array}{lll}
\infty & \text { if } \quad \mathrm{r} \leq \mathrm{R} \\
U_{0} & \text { if } \quad \mathrm{R}<\mathrm{r}<\mathrm{R}+\delta \\
0 & \text { if } \quad \mathrm{r}>\mathrm{R}+\delta
\end{array}\right.
$$

$R$ being the radius of the NP.
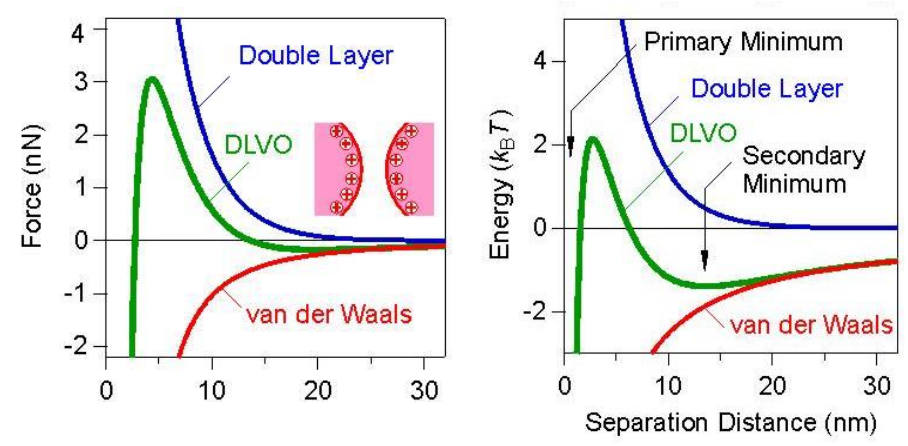

FIG. 7. Typical profile for a pairwise DLVO potential resulting from attractive van der Waals and repulsive double layer interactions. The two figures display a DLVO potential with one or two minima according to the van der Waals range [112].

\section{Particles with interaction in binary mixture}

Colloidal particles, due to their size, are often characterized by effective interactions whose range is significantly smaller than the particle diameter. The use of standard effective potential based on point charge is still debated. Colloidal stability requires that the repulsion and entropic contribution between the suspended particles is greater than the attractive dispersion force. When particles are surrounded (or not) by ligands the interactions are limited to (unretardated) van der Waals and electrostatic double layer interactions (the so-called DLVO approximation $[10,106,107])$. van der Waals forces dictate the profiles at large and small distances, while the double layer force dominates the intermediate distances. Other interactions are under consideration [108], including osmotic repulsion for capped particles with ligands (the repulsive forces due to overlapping of the long-chain capping material covering the metal nanoparticles and their interactions with the surrounding solvent molecules), steric repulsion, electrostatic interaction, hydrogen bonding, magnetic interaction, etc. All of them are related to a decomposition of pairwise potentials with a more or less extent as depicted in Fig. 7. Notice that a theoretical model including attractive forces between two sets of particules has provided recently complete phase diagrams for 2D and 3D systems [109], which are different from those with the well-known repulsive hard-sphere model [110, 111]. As mentioned previously, van der Waals forces promote a dense packing in fluid phase, other forces promote a dense packing in crystalline phase. For that purpose, the addition of co-solute 


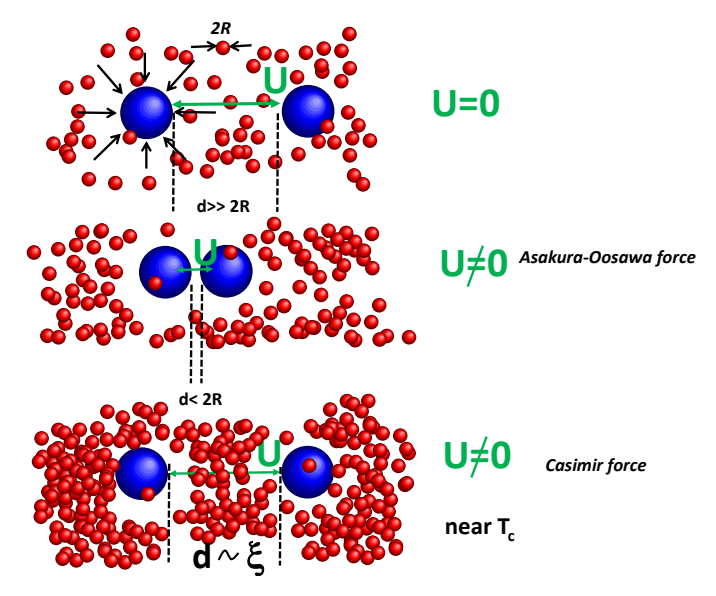

FIG. 8. Three configurations for a binary fluid, (a) the distance between the colloid particles is large compared to the co-solute particle, there is no additional force. (b) The distance is less than the diameter of the cosolute particle, due to the entropic term and sterinc hindrance, a net interaction is observed and described by Asakura-Oosawa potential [113]. (c) Near the criticality, when the coherence length $\xi$ is large enough, one observes an interaction given by a CasimirdeGennes-Fisher formalism.

particles allows manipulating and controling the phase behaviour of colloids by means of effective interactions (depletion) ??. Co-solute properties can be exploited to create new types of effective interactions among colloids. Far away the criticality, when the two colloids are at distances larger than $2 R$ (see Fig. 8a) the net pressure on each colloid is zero, the interactions are limited to DLVO. When the two colloids are closer than $2 R$ a "depletion" region appears between the two particles (see Fig. 8b). The mismatch of the pressure inside and outside the colloids gives rise to a non-zero balance. This force can be calculated analytically within Asakura-Oosawa model $[113,114]$. When the solvent correlation length becomes of the order of the two colloid particles distance (near the critical temperature), an effective force appears due to Casimir-deGennes-Fisher force (see Fig. 8c).

\section{External driving force}

There are few ways for driving or controling growth by external forces including magnetism [115], electrical field, laser beam, etc. Collino et al. [116] showed that acoustically-driven assembly can be exploited to achieve tunable patterning of anisotropic particles (Fig. 9).

Korda et al. [117] drive 2D monolayer of colloidal spheres by hydrodynamic forces into a large array of holographic optical tweezers. Jensen et al. studied the crystallisation after natural sedimentation under gravity and rapid deposition under ultracentrifugation. The crystallization depends strongly to the structure of the template (substrate). In this manner, they obtained rapid growth of large and defect free colloidal fcc crystals onto fcc(100)

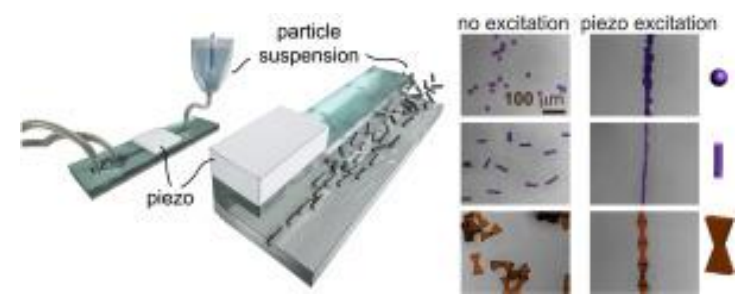

FIG. 9. Particle alignment via acoustophoresis. (Left) Schematic illustration. (Right) Micrographs showing random alignment of (top to bottom): glass microspheres, glass microrods, SU-8 bowties, and SU-8 bricks, when there is no acoustic excitation. Strong particle alignment and packing with acoustic excitation (for details see the original paper [116]).

tremplates. By contrast, fcc(111) template favours disordering under sedimentation.

\section{NPS SYNTHESIS}

\section{A. Strategy}

Two classes of elementary building-blocks (called NPs) are under consideration:

- Faceted NPs (nanocrystals): these smaller blocks, obtained through chemical and physical methods, require a selective functionalization of their oriented surface (using organic ligands, DNA fragments, etc).

- Micro (and nano) patchy particles: obtained by chemical engineering (polymerization) from modified spherical colloids to generate anisotropic particles [118].

It must be noted that the size of the considered particles ranges from few $\mathrm{nm}$ to microns and questions the validity of the models developed in the literature.

\section{B. Nucleation of colloidal crystals: theory}

The nucleation of a solid phase in a colloidal suspension has been treated since a long time using the classical nucleation theory from Volmer [119] and Becker-Dring [120] for atomic systems. This theory works relatively well for colloids that behave as hard spheres systems (HS) [121]. 


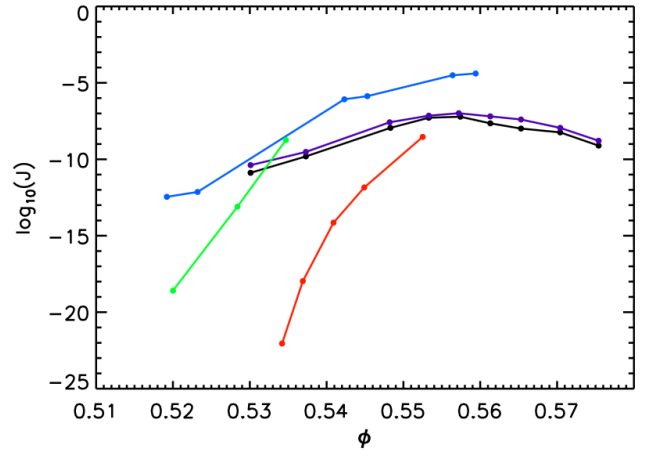

FIG. 10. Nucleation rate of hard spheres as a function of the filling fraction $\phi$ observed in experiments: maximum (purple curve) and average nucleation rates (black curve) from [122] and from [123] (blue curve) and in simulations: monodisperse (red curve) and polydisperse hard spheres from [124]. (From [121]) [112]

The total free Gibbs energy variation to form a spherical crystallite of radius $R$ is

$$
\Delta G=\frac{4}{3} \pi R^{3} \rho \Delta \mu+4 \pi R^{2} \gamma
$$

where $\rho$ is the density of the solid, $\Delta \mu$ is the difference of chemical potential between the solid and the liquid and $\gamma$ the solid-liquid interfacial energy. The first term corresponds to the formation of the solid nucleus and the second term to the creation of the surface of the nucleus. The maximum of the curve corresponds to the critical nucleus (after this size the nucleus grows spontaneously). The energy of the critical nucleus is:

$$
\Delta G_{\mathrm{crit}}=\frac{16 \pi}{3} \frac{\gamma^{3}}{(\rho|\Delta \mu|)^{2}} .
$$

The nucleation rate per unit volume is:

$$
J=\kappa \exp \left(\frac{-\Delta G_{\text {crit }}}{k_{\mathrm{B}} T}\right),
$$

where $\kappa$ is the kinetic prefactor, $k_{\mathrm{B}}$ the Boltzman constant and $T$ the temperature. Taking Eq. 14, the nucleation rate becomes

$$
J=\zeta \exp \left[\frac{-16 \pi \gamma^{3}}{(\rho|\Delta \mu|)^{2}}\right] .
$$

Figure 10 shows nucleation rate for experimental system and simulation with hard sphere (HS) systems. The supersaturation is obtained by cooling which increases the volume fraction $(\phi)$ in the condensed phase. The maximum of the nucleation rate is obtained close to the melting point which is at $\phi_{m}=0.545$ for a HS system. The agreement between experiment and simulation is qualitatively good. However, some quantitatively discrepancies appear between experiments and simulations. These disagreements can be due to the fact that the nucleus shape is different from a sphere due to the crystalline structure of the bulk material. In fact, experiments and simulations have shown that the nuclei can be flat [125] or multiple twins close to a decahedron [126]. Crystallization of DNA coated microspheres has been obtained experimentally only recently [127]. The DNA colloidal particles behave like uncovered colloid, a nucleation stage is observed before growth which is compatible with classical nucleation theory [127]. However the window to observe well-ordered crystallization is quite reduced [127, 128]. Particle with DNA strands attached at well-defined positions (patchy particles) form directional bonding that opens the way to the formation of non-compact (fcc or bcc) structures (see Sec. IX). In this case, computer simulations show that the nucleation barrier significantly changes with the number of patches [129]. In the case of nanoparticles, few experimental works on nucleation have been published. The self-assembly of gold nanoparticles (4.9 nm) coated by ligands has been studied by dynamic light scattering. The size of the nuclei as a function of temperature has been well fitted with the classical nucleation theory [130].

\section{Mechanism of growth in the framework of the La Mer model}

The key idea of separating the nucleation stage and growth process in time is often used to obtain nearly mono disperse particles. Within this hypothesis, one introduces the concept of the self focusing regime. This cannot be achieved in nucleation growth in free gas phase since the high rate of growth leads to a growth time in the same order of magnitude than the nucleation one. This is not the case in liquid (colloidal) phase where one observes a burst of nuclei formation in a short period, an initial fast rate of growth of these nuclei and a slow rate of growth leading to a long growth period compared to the nucleation period (often referred to Lamer's mechanism [132]). Now, assume that the average radius of the particles is $R$. The bulk liquid phase (media) is considered to have a uniform supersaturated monomer concentration, $C_{B}$, while the monomer concentration at the particle interface noted $C_{i}$ is depleted, $C_{R}$ the solubility of the particle with a radius $R$ (Fig. 11) which is related to $C_{\infty}$ by the Gibbs-Thomson relationship given by [133]:

$$
C_{R}=C_{\infty} \exp \left(\frac{2 \gamma_{\mathrm{int}} v}{R k_{\mathrm{B}} T}\right)
$$

$\gamma_{\text {int }}$ is the interfacial energy, $v$ the molar mass, $C_{\infty}$ the concentration for a flat surface. One obtains the critical radius (see Fig. 11)

$$
R_{\text {crit }}=\frac{2 \gamma v}{k_{\mathrm{B}} T \log S} .
$$




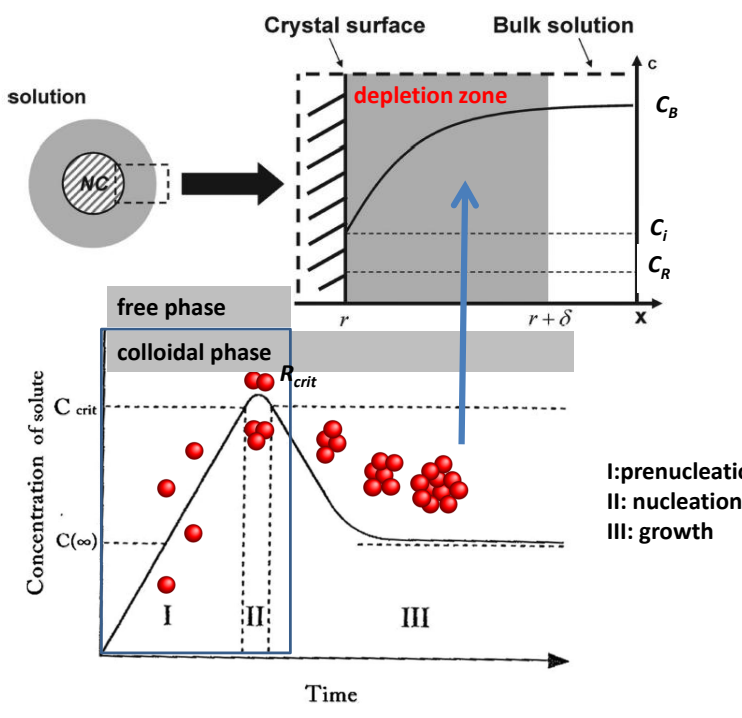

FIG. 11. Schematic illustration of diffusion layer showing the depletion zone around a cluster with infinite radius (flat surface). The gradient induces diffusion matter allowing cluster growth. This mechanism is given by the Lamer diagram (bottom) showing the three regions, prenucleation, rapid nucleation and growth. In free phase, cluster nucleation and growth in the same I/II regions. The well separated regions permits the self focusing regime (see text). After the original figure in [131].

This critical radius corresponds to the minimum size at which a particle can survive in solution without being redissolved.

The Gibbs-Thomson law states that the solubility is not independent of the size. Both the particles and media are fluids, the solute atoms diffuse to the spherical particles under steady-state condition. The growth originates from the concentration gradients at the vicinity of the surface. The concentration at the surface of particles in equilibrium with larger particles is lower than that with smaller particles as depicted in Eq. 17. Atoms in the media flow through the concentration gradients both from the surface of the smaller particles to media and from the media to the surface of larger particles, this is the so-called Ostwald ripening (i.e. the diffusion of mass from regions of high interfacial curvature to regions of low interfacial curvature). The process occurs by the growth of large particles at the expense of smaller ones which dissolve. One can introduce a critical radius $R_{\text {crit }}$ where particles with $R>R_{\text {crit }}$ grow and particles with $R<R_{\text {crit }}$ shrink (we assume that $R_{\text {crit }}$ is a constant). The flux of monomers, $J$ passing through a spherical surface with radius $x(x \ll \delta$ where $\delta$ is the depletion zone within the diffusion layer) is given by Fick's first law $[134,135]$. At the steady state,

$$
J=4 \pi D R\left(C_{B}-C_{i}\right)
$$

This flux is compensated by the consumption rate of the monomers near the surface

$$
J=4 \pi R^{2} k_{d}\left(C_{i}-C_{R}\right)
$$

where $k_{d}$ is the rate constant, $D$ the diffusion coefficient. Assuming that $d R / d t=J v / S$ with $S=4 \pi R^{2}$, the master equation for the $R$ time dependence in the general case writes

$$
\frac{d R}{d t}=\frac{D v / R\left(C_{B}-C_{R}\right)}{1+D / k_{d} R} .
$$

$C_{B}$ and $C_{R}$ are given by Eq. 17 and

$$
C_{B}=C_{\infty} \exp \left(\frac{2 \gamma_{\mathrm{int}} v}{R_{\mathrm{crit}} k_{\mathrm{B}} T}\right) .
$$

One assumes $\frac{2 \gamma_{\mathrm{int}} v}{k_{\mathrm{B}} R_{\mathrm{crit}} T} \ll 1$ then

$$
\frac{d R}{d t}=\frac{2 \gamma_{\mathrm{int}} v^{2} C_{\infty}}{k_{\mathrm{B}} T\left(\frac{1}{D}+\frac{1}{k_{D} R}\right)} \frac{1}{R}\left(\frac{1}{R_{\mathrm{crit}}}-\frac{1}{R}\right)
$$

Two asymptotic behaviours are under consideration, namely $D \ll k_{d} R$ and $D \gg k_{d} R$. The first case $D \gg k_{d} R$ called reaction-limited growth where the growth rate is limited by the surface reaction of the monomers is derived from Eq. 23

$$
\frac{d R}{d t} \propto \frac{1}{R}\left(\frac{R}{R_{\text {crit }}}-1\right)
$$

Assuming that size distribution is centered around $\bar{R}$ with a dispersion $\Delta R / \bar{R}$. Previous equation writes (assuming that the critical radius $R_{\text {crit }}$ is a constant)

$$
\frac{d \Delta R}{d t} \propto \frac{\Delta R}{\bar{R}^{2}}
$$

The dispersion increases with time leading to a broadening with mean size increase.

The second case $D \ll k_{d} r$ called diffusion limited growth regime states that the particle growth is essentially controlled by the diffusion of the monomers to the surface. This regime was first described by Ostwald and developed by Lifshitz, Slyozov and Wagner (LSW) [134136]. Equation 23 writes

$$
\begin{gathered}
\frac{d R}{d t} \propto \frac{1}{R^{2}}\left(\frac{R}{R_{\text {crit }}}-1\right), \\
\frac{d \Delta R}{d t} \propto \frac{\Delta R}{R^{2}}\left(\frac{2}{\bar{R}}-\frac{1}{R_{\text {crit }}}\right) .
\end{gathered}
$$

When clusters grow and reach $2 R_{\text {crit }}, d \Delta R / d t$ becomes negative. Then, the size distribution narrows within increasing size, this is the self-focusing regime. Nucleation regime is intermediate between the two diffusion limited growth and reaction-limited growth regimes. However, 
the monodispersity needs to approach the diffusion limited growth regime: smaller particles will grow more quickly than larger particles, leading to a narrowing of the size distribution. Another way to reach a self focusing regime needs nucleation of binary or ternary compounds with a core shell structure [137-139]. In certain conditions smaller (binary/ternary) particles grow at expense of the larger ones, this is the digestive ripening which is the inverse of Ostwald ripening. Core-shell structures are an interesting field as long self-assembly of self-limiting monodisperse superlattices can be obtained from polydisperse nanoparticles [140]. In this case, the self-limiting growth process (i.e. self-focusing regime) is yet governed by a balance between electrostatic repulsion and van der Waals attraction, which is aided by the broad polydispersity of the nanoparticles.

\section{SUPRACRYSTAL BASED ON SPHERICAL (ISOTROPIC) PARTICLES}

\section{A. Size dispersity}

In the literature, a common sentence is "NPs are sufficiently mono-disperse that they form well-ordered 2-D hexagonal lattice or close-packed supracrystals". The introduction of spherical NPs with different sizes into the otherwise mono dispersed sample of NPs may lead to disordering and phase segregation during the crystallization process. It may seem intuitive at first glance, this idea that somehow a perfect long range order needs identical spheres packing. In fact, it is not true since a collection of different spheres can form crystals (if we add smaller spheres to pack the vacancy of a fcc packing, we can increase the density further). However, this specific case corresponds to spheres with well-defined ratios and will be discussed later (the so called "the packing together of many discretely sized particles" [141]). If we consider a Gaussian size distribution $\sigma$ (the dispersion of the packing of continuous particle size distributions [141]), which is realistic for a nucleation/growth process, the long range order rapidly vanishes with $\sigma$. This is difficult to make a criterion giving the upper limit of $\sigma$ for a perfect crystallization. We just define an upper theoretical limit: long range order is wholly disrupted once the distribution of NPs sizes reaches a value of dispersion $\sigma=0.27$ (defined as the standard deviation of the distribution of radii divided by its mean value within a triangular distribution)[142]. This is the upper limit where there is no way of solving phase transition (i.e. crystallisation). It is found experimentally that colloidal systems with a polydispersity $\sigma$ greater than about 0.10 do not crystallize on an experimentally accessible timescale at any concentration [143]. More recently, it has been shown that fractionation into several solid phases can occur with possible coexistence of several solids with a fluid phase (Fig. 12) [144]. Crystallization in a single structure needs very low polydispersity as depicted in Fig. 12 [145].

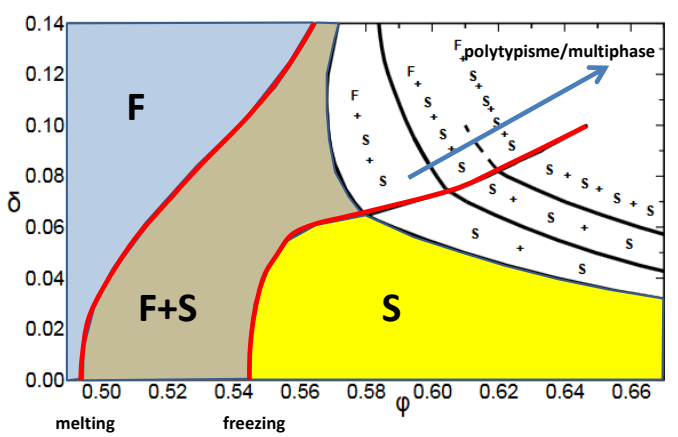

FIG. 12. Phase diagram for poly-disperse hard spheres with a triangular size distribution. In each region the nature of the phase(s) coexisting at equilibrium is indicated (F: fluid, S: solid). The yellow region corresponds to the crystallisation in one component. Phase coexistence between solid and liquid as depicted in Fig. 13 appears at higher compacity with polydispersity increase [145].

There is no way to observe a phase transition (crystallisation) when the variance is higher than 0.27 (upper limit) and probably limited to 0.1 (Fig. 12).

\section{B. Topology}

A 3D periodic lattice needs a short and a long range order. Supracrystal can be considered as a sphere packing problem. From the topology point of view, the long range order needs two conditions: monodispersity and dense packing. The first is related to the synthesis of the NPs, the second is related to the attractive or repulsive interaction between the NPs (crystallization). This latter can be viewed as a first order transition according to Landau's theory and is related to a fluid-crystalline phase transition where entropy drives crystallization [146] .

\section{Crystallization}

The volume fraction filled by the solid objects in random close packing cannot exceed a density limit of $63.4 \%$ [148] while in dense packing (crystal like) this value is $0.74 \%$ according to Hales-Kepler conjecture [103]. Hardsphere system can freeze [146] with no attractive inter NPs interactions as depicted in the phase diagram (Fig. 13). The coexistence between solid and liquid-like is defined in the range of density $0.494-0.545$. The thermodynamical stable system corresponds to the fcc crystal branch. However, kinetics dictates the crystallisation or not by the comparison of the time scale for the non-equilibrium to metastable equilibrium transition compared to the time scale related to the transition from 


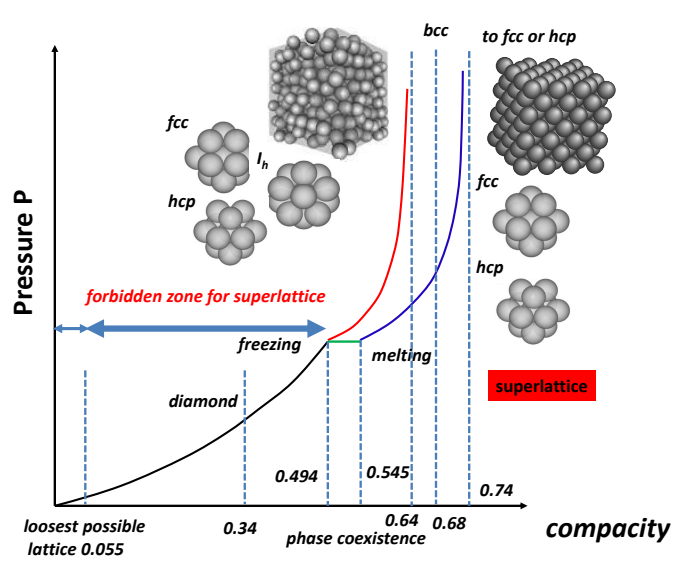

FIG. 13. Phase diagram for the sphere packing problem showing the phase coexistence without interaction (hard sphere model) [146]. The stable branch gets fcc structure while metastable structure gets the random compact packing hcp. fcc or hcp with associated polytypes (fcc, hcp or combinations just differ by entropy energy) need defect free structures. Icosahedral symmetry which is locally more compact introduces topological defects hindering a superlattice construction. This is the way to obtain hcp metastable structures. Below the freezing, no superlattice (diamond for example) can be obtained with a standard route (pairwise interaction potential). Note that no expected superlattice exists below the loosest packing limit 0.055 [147].

the metastable to the stable ordered branch. In the superlattices where some interactions between NPs take place, the problem quickly becomes intractable. We select two cases under consideration: attractive or repulsive isotropic pairwise potential. If the attractive potential is isotropic and limited at short distance (first neighbors like van der Waals bonding), the criterion is the most local packing configuration [149]. According to Roger's conjecture [102], the maximum possible local packing density is 0.78 a little bit more than fcc structure. This value is conjectured to mimick the icosahedral packing at the first neighbouring shell (13 atoms, see Fig. 13). Since fivefold symmetry is incompatible with long-range order, pentagons entail an energetic barrier to rearrangement thus hindering crystallization and allowing the fluid to persist at temperatures well below the freezing point. Conversely, a soft repulsive potential decreases the compacity and then crystallization occurs in bcc structure which is lower in compacity 0.68 than fcc. The crystallization is a subtle balance between the compacity, the nature of the interaction potential (many body, non isotropic, etc.) and the kinetics (solvent evaporation time) [150]. The phase diagram depicted in Fig. 13 reveals that superlattice with a low compacity (below 0.494 ) cannot be synthesized without the help of external ingredients as long the crystallization cannot be reached. The aim of this review is to discuss these strategies in the following.

At this stage, the synthesis of non dense superlattices
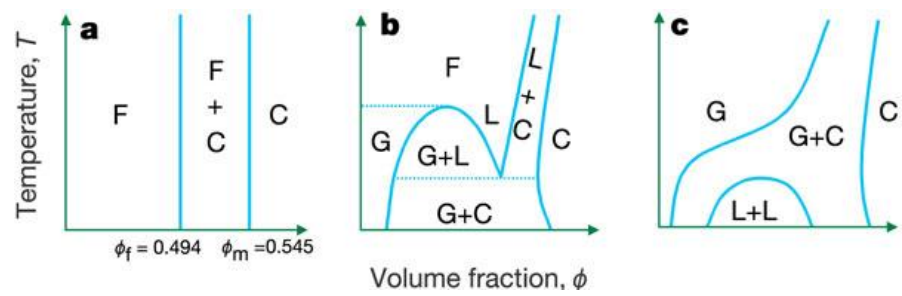

FIG. 14. Colloidal phase diagram: (a) purely hard-sphere system, (b) long-range attraction system, and (c) short-range attraction system (from [151]), G gas, L liquid, F fluid, C crystal.

through spherical non interacting NPs is not expected. Other scenarios must be considered.

\section{Particles with interaction}

In this section, we discuss the case of packing structures. The phase diagram for a fluid of hard spheres without interaction is depicted in Fig. 14a (see also Fig. 13). The addition of long-range attractions results in threephase equilibria, with a triple point and phase line between liquid and gas ending in a critical point leading to the much complex phase diagram depicted in Fig. 14b [151]. Likewise, for short-range attractions (Fig. 14c), equilibrium between gas and crystal is observed, but the gas-liquid equilibrium becomes metastable. This underlines the complexity of the phase diagram according to the type of interaction between particles. According to Fig. 13, for hard sphere without interaction, the critical density $\rho_{c}$ of the phase coexistence where both fluid and solid can coexist until the melting point is $\phi_{c}=0.545$. This density depends on the interaction between spheres (particles). Table II reports critical density for van der Waals interaction and adhesive hard-sphere potential (AHS) defined by a square-well potential $U(r)$ [152].

Contrary to vdW interaction which is attractive [153], AHS potential supposes that the structure is dominated by the hard repulsion part of the intermolecular potential. Reading the table shows that the potential tunes the boundary between the phase coexistence. Note that for $\delta<0.25$ gas-liquid separation becomes metastable with respect to the fluid-solid equilibrium. 
TABLE II. Critical point parameters for the square-well potential fluid for width $\delta$ (in units of $R$ ) [152] and vdW potential [153].

\begin{tabular}{|l|l|l|l|l|l|l|l|l|}
\hline$\delta$ & 0.0 & 0.005 & 0.01 & 0.02 & 0.03 & 0.04 & 0.05 & 0.10 \\
$\phi_{c}$ & 0.545 & 0.542 & 0.540 & 0.538 & 0.530 & 0.522 & 0.513 & 0.478 \\
\hline$\delta$ & 0.20 & 0.30 & 0.40 & 0.50 & 0.60 & 0.70 & 0.80 & - \\
$\phi_{c}$ & 0.421 & 0.376 & 0.339 & 0.310 & 0.287 & 0.272 & 0.263 & $0.25(\mathrm{vdW})$ \\
\hline
\end{tabular}

\section{SUPRACRYSTAL BASED FROM NON-SPHERICAL PARTICLES: THEORY AND COMPUTER SIMULATION OF PARTICLE SELF-ASSEMBLY}

\section{A. Theory of self-assembly}

For a long time humanity was questioning about the compact assembly of identical objects. As we have seen before, Kepler was interested in the maximal filling of space by spheres. He conjectured that the packing fraction was $\pi / 18^{1 / 2}=0.74048 \ldots$ which corresponds to a fcc structure. However, we have no exact answer for other congruent convex objects besides the trivial cases of cube and truncated octahedron that fill the space. The conjecture of Ulam reported by M. Gardner in 1972 predicts that the maximum packing fraction for an assembly of convex objects is always larger than those of spheres [154]. Tetrahedra, which represent an important class of NPs [155], are an interesting case. The regular packing of tetrahedra with $\phi=2 / 3$ and an irregular arrangement with $\phi=0.72$ have been predicted [102] while the densest Bravais lattice of tetrahedra corresponds to $\phi=18 / 49=0.367 \ldots$ Experimentally, a packing estimated to $\phi \simeq 0.59$ was obtained for CdSe tetrahedra [155]. These results are clearly in contradiction with Ulam's conjecture. However experiments with tetrahedral dices later showed a maximum compactness with $\phi \simeq 0.75$ but the dice were non perfect tetrahedra (the vertices and edges were rounded) [156]. Later, Chen founds a periodic packing of tetrahedra with $\phi=0.7786$ [157] that clearly is larger than those of spheres. The Ulam conjecture was verified. In 2009, Torquato and Jiao [158] studied the densest organization of the 5 Platonic solids (tetrahedron, icosahedron, dodecahedron, octahedron, cube) and the 13 Archimedean solids (the Archimedean solids are polyhedrals composed of two or more regular polygons meeting in identical vertices): truncated tetrahedron (A1), truncated icosahedron (A2), snub cube (A3), snub dodecahedron (A4), rhombicosidodecahedron (A5), truncated icosidodecahedron (A6), truncated cuboctahedron (A7), icosidodecahedron (A8), rhombicuboctahedron (A9), truncated dodecahedron (A10), cuboctahedron (A11), truncated cube (A12), truncated octahedron (A13), which are represented in Fig. 15. They found theoretically a formulation for the upper bound of the maximum density of packing

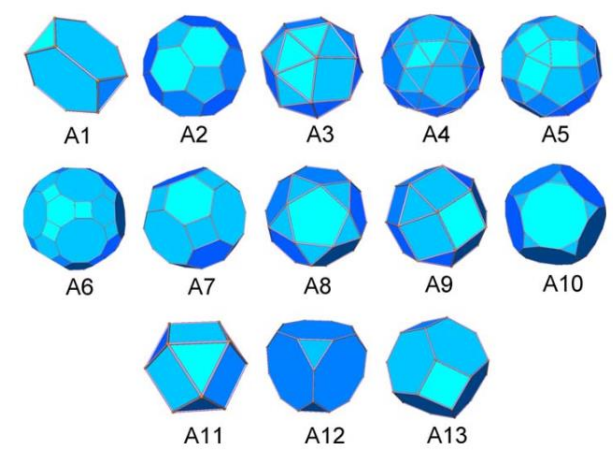

FIG. 15. The 13 Archimedean solids: truncated tetrahedron (A1), truncated icosahedron (A2), snub cube (A3), snubdodecahedron (A4), rhombicosidodecahedron (A5), truncated icosidodecahedron (A6), truncated cuboctahedron (A7), icosidodecahedron (A8), rhombicuboctahedron (A9), truncated dodecahedron (A10), coboctahedron (A11), truncated cube (A12), truncated octahedron (A13). (From [158])

for nonspherical particles:

$$
\phi_{\max }^{u}=\min \left(\frac{V_{p} \pi}{V_{s} 18^{1 / 2}}, 1\right),
$$

where $V_{p}$ is the volume of a d-dimensional particle and $V_{s}$ is the volume of the largest sphere that will be inscribed in the nonspherical particle. In the same paper [158], they calculate by numerical simulations the maximum packing $\left(\phi_{\max }\right)$ for Platonic solids. The value of $\phi_{\max }, \phi_{\max }^{L}$ (the optimal lattice packing) and $\phi_{\max }^{U}$ obtained for Platonic and Archimedean solids are shown in Tab. III. It is interesting to note that for the tetrahedron $\left(\phi_{\max }=0.8563\right)$ the densest packing obtained in the simulations has no long range order unlike for the other Platonic solids for which the maximum density corresponds to Bravais lattices. Torquato and Jiao conjectured that the densest packing of platonic and Archimedean solids with central symmetry are given by the corresponding densest lattice packing [158]. Tetrahedron is the only non-centrosymmetric Platonic solid as the truncated tetrahedron for the Archimedean solids which presents a much higher density $(\Phi=0.9951)$ than the corresponding densest lattice (0.6809). Several authors have also studied the densest packing of tetrahedral by computer simulations. They found maximum packing fraction of 0.8503 [164], 0.8547 [165], 4000/4671 = 0.8563 $[159,160]$. The last calculations constructed the lattice from an analytic formulation. Cube is the only Platonic solid that completely fill space but in combining octahedra and tetrahedra it is also possible [166]. Numerical methods have been recently elaborated to study regular packings of irregular nonconvex particles [167]. In Tab. III, we give the maximal density obtained by numerical simulations for Platonic and Archimedean solids. 
TABLE III. Theoretical results of the densest organization for the 5 Platonic solids, the 13 Archimedean solids and the rhombicdodecahedron (Catalan solid). $\phi_{\max }^{L}, \phi_{\max }^{U}$, and $\phi_{\max }$ are the density for the optimal lattice, the upper-bound value and the maximum value observed in the simulations, respectively. Tetrahedron (T), Icosahedron (I), Dodecahedron (D), Octahedron (O), Cube (C), Truncated tetrahedron (A1), Truncated icosahedron (A2), Snub cube (A3), Snub dodecahedron (A4), Rhombicicosidodecahedron (A5), Truncated icosidodecahedron (A6), Truncated cuboctahedron (A7), Icosidodecahedron (A8), Rhombicuboctahedron (A9), Truncated dodecahedron (A10), Cuboctahedron (A11), Truncated cube (A12), Truncated octahedron (A13), Rhombic dodecahedron (RD). Minkowski lattice (Ml), body-centered cubic (bcc), simple cubic (sc), face-centered cubic (fcc).

\begin{tabular}{|l|l|l|l|l|l|}
\hline Shape & Lattice & $\phi_{\max }^{L}$ & $\phi_{\max }^{U}$ & $\phi_{\max }$ & reference \\
\hline & & $\begin{array}{l}\text { Platonic } \\
\text { Solids }\end{array}$ & & & \\
\hline T & - & 0.3673 & 1 & 0.8563 & {$[158-160]$} \\
I & - & 0.8363 & 0.8934 & 0.8363 & {$[158]$} \\
D & $\beta-M n(A 13)$ & 0.9045 & 0.9811 & 0.9045 & {$[158,161]$} \\
O & Ml & 0.9473 & 0.9811 & 0.9473 & {$[158]$} \\
C & sc & 1 & 1 & 1 & {$[158]$} \\
\hline & & Archimedean & & & \\
& & Solids & & & \\
\hline A1 & & 0.6809 & 1 & 0.995 & {$[161,162]$} \\
A2 & & 0.7849 & 0.8356 & 0.7849 & {$[158]$} \\
A3 & & 0.7876 & 0.9349 & 0.7873 & {$[158]$} \\
A4 & & 0.7886 & 0.8554 & 0.7886 & {$[158]$} \\
A5 & & 0.8047 & 0.8359 & 0.8047 & {$[158]$} \\
A6 & & 0.8272 & 0.8973 & 0.8772 & {$[158]$} \\
A7 & & 0.8493 & 0.8758 & 0.8493 & {$[158]$} \\
A8 & & 0.8647 & 0.9380 & 0.8647 & {$[158]$} \\
A9 & & 0.8758 & 1 & 0.8758 & {$[158]$} \\
A10 & & 0.8977 & 0.9738 & 0.8977 & {$[158]$} \\
A11 & fcc & 0.9183 & 1 & 0.9183 & {$[158,163]$} \\
A12 & sc & 0.9737 & 1 & 0.9737 & {$[158]$} \\
A13 & bcc & 1 & 1 & 1 & {$[158]$} \\
\hline & & Catalan & & & \\
& & Solids & & & {$[161]$} \\
\hline RD & fcc & 1 & 1 & 1 & \\
\hline
\end{tabular}

We add also the case of the rhombic dodecahedron which is a space filling Catalan solid for which self-assembly has been experimentally studied.

\section{B. Computer simulation}

Computer simulations have been extensively used in the recent years to study the self-assembly of particles. Glotzer et al. have studied the phase diagram of 145 convex polyhedra [161] (Fig. 16). Other groups have tackled the case of crystal packings of ellipsoids and rods $[168,169]$ As only hard particles interactions are considered, the simulation can be made on a large number of particles. Packing efficiency increases the alignment of flat facets that maximizes the entropy and then the stability of the assembly. These simulations used Monte Carlo methods.

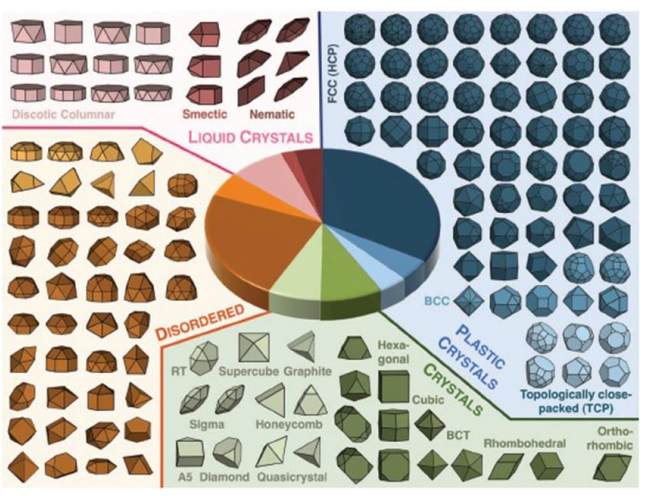

FIG. 16. Different organizations of 145 polyhedra from Monte Carlo simulation. (From [161]). Four types of organization are observed: crystal, plastic crystal, liquid crystal and disordered glass. For the crystalline structures 5 Bravais lattices were observed: cubic, hexagonal, body centered tetragonal, rhombohedral and orthorhombic. Quasicrystalline order has been also observed in the simulations for tetrahedra [164]. Dijkstra and collaborators have obtained a complete phase diagram for the self-assembly of truncated cubes [170]. By using molecular dynamics it is possible to introduce other interactions than hard particles that makes the simulations more realistic comparisons to the experiments [171]. By using large scale molecular dynamics with coarse-grained methods, it is possible to take also account the solvent in the simulation $[172,173]$.

\section{Comparison between theoretical models and experiment: case of polyhedron particles}

Table IV gives experimental observations for selfassembly of polyhedron nanoparticles in the size range $10-300 \mathrm{~nm}$.

\section{Tetrahedra}

PbSe tetrahedra with a size of $10 \mathrm{~nm}$ self-organize in an orthorhombic Bravais lattice [155]. No face to face contact but rather edge to edge contact is observed on the contrary of the simulations [158-160]. The arrangement of tetrahedra is less dense than predicted theoretically ( $\Phi \simeq 0.59$ taking into account the ligands). The discrepancies between theory and experiments can be solved if we introduce other interactions than in the case of hard particles which are stabilized by maximization of entropy. These new interactions are due to the ligand-ligand interaction which is dependent of the nature of the solvent. In the case of a bad solvent (or vacuum) the interaction between edges is attractive while the interaction between the facets is weakly repulsive and in the case of good solvent the strong repulsion between ligand on the facets is also favorable to vertex to vertex contact [155]. 


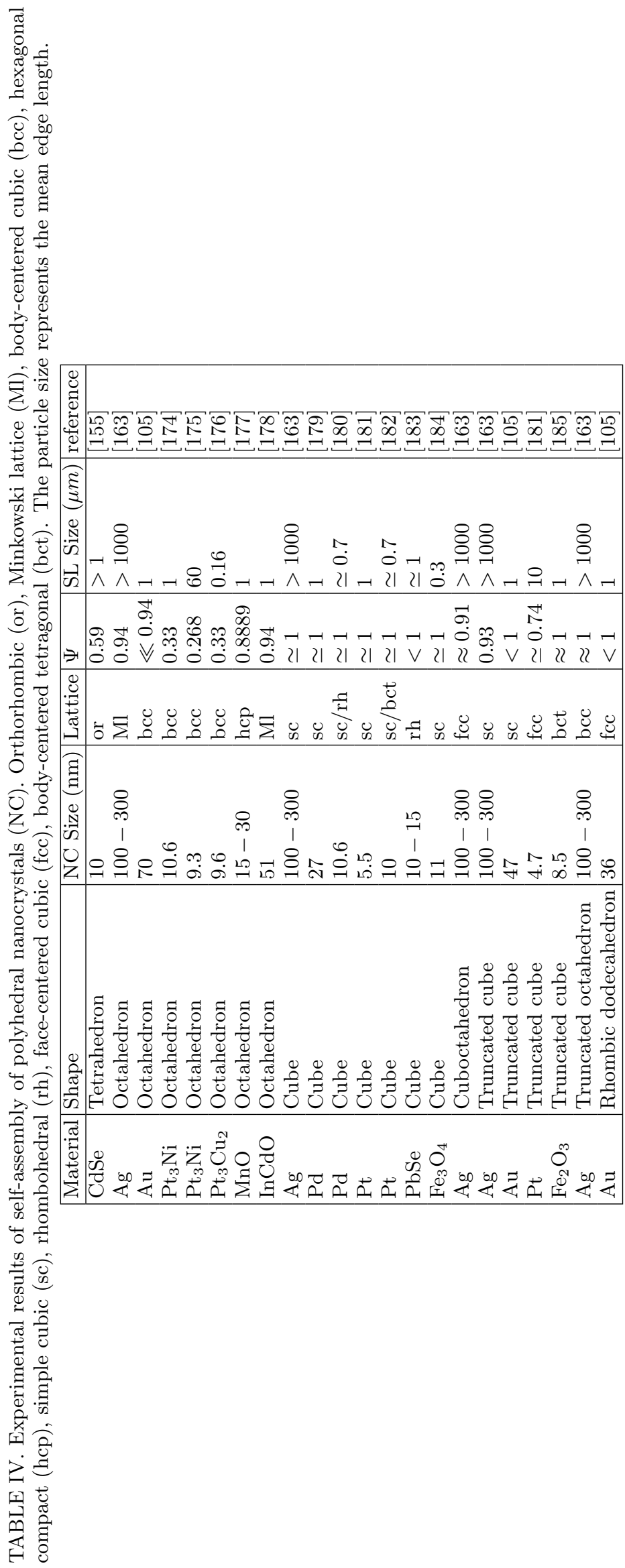

\section{Octahedra}

The self-assembly of octahedral NPs (size from 9 to 300 $\mathrm{nm})$ from different materials $\left(\mathrm{Ag}, \mathrm{Au}, \mathrm{Pt}_{3} \mathrm{Ni}, \mathrm{Pt}_{3} \mathrm{Cu}_{2}\right.$, $\mathrm{MnO}, \mathrm{InCdO})$ has been studied $[105,163,174-178]$. They self-assemble in a bcc lattice except for Ag [163] and $\mathrm{InCdO}[178]$ which form a Minkowski lattice (Ml) and $\mathrm{MnO}$ [177] which forms a hcp lattice. The prediction from theory was the $\mathrm{Ml}$ which corresponds to a very dense arrangement $(\Phi=0.94)$. For the bcc lattice the packing is much less dense $(\Phi=0.33)$ while for the hcp lattice the filling factor $(\Phi=0.88)$ is a little smaller than for the Ml. In the dense structures (Ml, hcp) a high number of face to face contact are observed which is explained by a maximization of entropy. For large particles $(50-300 \mathrm{~nm})$ the particles follow the structure (Ml) predicted by the theory for hard particles which means that entropic directional forces are the most important interaction which favors face to face contacts. Small particles (around $10 \mathrm{~nm}$ in size) are organized in the bcc structure which is not dense $(\Phi=0.33)$. In this structure the particles tend to form tip to tip contact. For this range of sizes, interactions other than hard particle ones become important. In particular the interactions between facets through adsorbed ligands are strongly repulsive and face to face contact is impossible. On the edges and vertices of the octahedra the ligand-ligand interaction between two particles is less repulsive which explains the preference for tip to tip contact. The $\mathrm{MnO}$ octahedra [177] lay within an intermediate size range and they self-organize in a dense structure $(\Phi=0.88)$ but less dense than for large particles $(\Phi=0.94)$. Gold octahedra with a size of $70 \mathrm{~nm}$ [105] should have a dense structure but they adopt the bcc structure. However this is a particular case. Indeed, surfactant (CTAB) is added in large quantity (much larger than the critical micellar concentration) forming micelles in the solution which creates depletion interactions [105]. Surfactant forms also a bilayer on the surface of the particles and since the CTAB molecule is charged, repulsive electrostatic interactions are present. Then, the distance between particles correspond to the situation where depletion and electrostatic forces compensate. By changing the concentration of surfactant or the ionic strength the distance between the particles can be tuned (in a limited range) [105].

\section{Cubes}

The self-organization of cubes of different materials $\left(\mathrm{Ag}, \mathrm{Pd}, \mathrm{Pt}, \mathrm{PbSe}, \mathrm{Fe}_{3} \mathrm{O}_{4}\right.$ ) in the size range 5 to $30 \mathrm{~nm}$ has been studied. In most cases the simple cubic structure which fills the space has been observed [163, 180182, 184] in agreement with Monte Carlo simulations with hard particles. The fact that the simple cubic (sc) structure can be formed even for small sizes is due to the cubic shape of the nanoparticles which induces a tetracoordination of the NPs corresponding to the most stable 
organization. However, if the NPs are not regular cubes the sc structure is no longer observed. When the 8 corners of the cubes are rounded, the particles self-assemble in a rhombohedral $(\mathrm{RH})$ structure as observed for $\mathrm{Pd}$ [180] and PbSe [183]. The continuous and reversible evolution of the structure of the NPs assembly has been observed for Pd cubes covered with docanethiol ligands which strongly interact with $\mathrm{Pd}$ via sulphur atom. The shape of the particles evolves as a function of the evaporation of the solvent from a perfect cube with rounding of the corners, then of the edges and eventually at the end the particles reach a spherical shape [180]. In the case of $10 \mathrm{~nm}$ Pt cubes different organizations have been observed depending on the nature of the solvent [182]. In toluene (aromatic) the sc structure is observed while in hexane (aliphatic) the body-centered-tetragonal structure (bct) is observed. This difference is due to a delicate balance between ligand-ligand and ligand-solvent interactions. The repulsion between ligands (oleylamine in this case) is stronger in hexane than in toluene then in the former case the bct structure allows to reduce the sum of the ligand-ligand interactions.

\section{Cuboctahedra}

The self-organization of perfect cuboctahedra has been studied only for large $(\simeq 200 \mathrm{~nm}) \mathrm{Ag}$ particles which form a fcc structure as expected from simulation with hard cuboctahedra [163].

\section{5. $\quad$ Truncated cubes}

The self-assembly of truncated cubes of $\mathrm{Ag}, \mathrm{Au}, \mathrm{Pt}$ and $\mathrm{Fe}_{2} \mathrm{O}_{3}$ in the size range $4.7-300 \mathrm{~nm}$ has been studied. Large Ag [163] and Au [105] particles form the sc structure expected from theoretical calculations. Particles, smaller than $10 \mathrm{~nm}$, form different structures. Pt particles of $4.7 \mathrm{~nm}$ self-organize in fcc structure [181]. The stability of the less dense fcc structure is explained by multipolar electrostatic interactions resulting from charge transfer between ligands and Pt cores [181]. The energy minimum is obtained when the (111) facets on the corners of the truncated cubes in a layer are shifted relatively to those of the adjacent layer. For $8.5 \mathrm{~nm} \mathrm{Fe}{ }_{2} \mathrm{O}_{3}$ truncated cubes a bct organization is found [185] instead of the expected sc one. The bct structure is explained by the anisotropy of the van der Waals attractive interaction induced by the (111) facets at the corners of the cube. Energetic calculation shows that the transition from sc to bct structure occurs when the degree of truncation increases.

\section{6. $\quad$ Truncated octahedra}

Large (around $300 \mathrm{~nm}$ ) silver particles with a truncated octahedron shape self-assemble in a bcc structure (Kelvin structure) as expected from calculations with hard polyhedra, the truncated octahedron like the cube is a space filling solid.

\section{Rhombic dodecahedron}

Gold rhombic dodecahedra [105] with a relatively large size of $36 \mathrm{~nm}$ self-assemble in a fcc structure as expected from calculations with hard particle interactions. All the (110) facets are aligned. However the facets are not in contact as expected from theory (rhombic dodecahedra fill the space), the measured gap size $(15.3 \mathrm{~nm})$ being larger than those expected from the ligand length. This large gap results from the competition between electrostatic and depletion forces.

Comparison between experimental results on the selfassembly of large neutral particles (typically larger $30-$ $300 \mathrm{~nm}$ ) show a good agreement with theoretical calculations for hard polyhedra. As mentioned later, this can be understood by the fact that for these sizes the van der Waals attraction is screened therefore the driving force for the self-assembly is the increase of entropy by maximizing the face to face contacts. These entropic forces are directional in contrast to the spherical colloids. Very dense packing with a filling factor close to one can be obtained. For small particles (typically smaller than 20 $\mathrm{nm}$ ) disagreements between simulations and experiment appear. In fact, small particles no longer can be considered as hard particles. Experimentally much less dense structures are observed. This is due to the strong repulsion between the facets of the nanoparticles arising from the interaction between ligands of two neighboring particles. For example tetrahedra or octahedra prefer tip-totip contacts instead of face-to-face contacts. When cubic particle are truncated, the appearance of (111) facets creates an axial anisotropy which gives rise to new structures like rh or bct. By changing the relative strength of attractive and repulsive forces between the particles the gap between particles can be adjusted.

\section{ANISOTROPY: BIMODAL DISTRIBUTION}

\section{A. Isotropic particles}

The low compacity can be obtained by using a combination of two nanoparticles with a bimodal distribution. As mentioned previously, the crystallization is in competition with the fluid phase. The packing in fluid phase (random close packing, rcp) for a bimodal distribution 


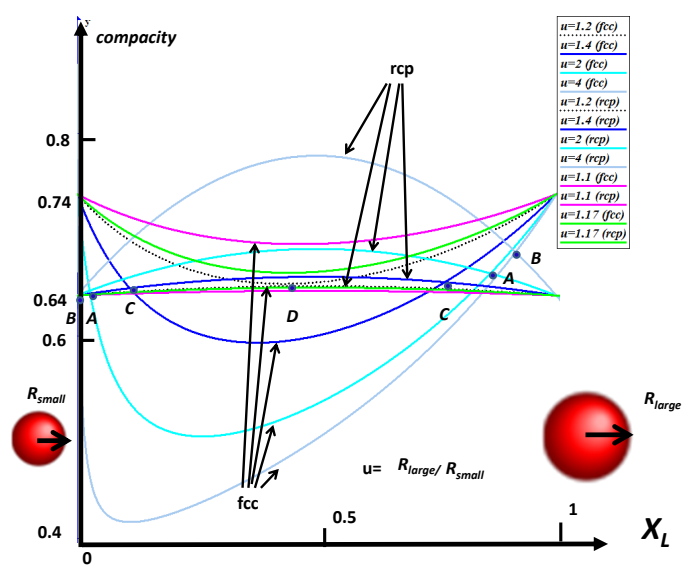

FIG. 17. Packing fraction in rcp and fcc structure for a set of parameters $u$ and $X_{L}$ derived from Eqs. 12 and 7. The stability is given by the maximum of compacity. The letters $\mathrm{A}, \mathrm{B}, \mathrm{C}$ and $\mathrm{D}$ show the crossover between rcp and fcc structures. Within the interval, $\mathrm{AA}, \mathrm{BB}, \mathrm{CC}$ and $\mathrm{DD}(\mathrm{D}$ is the critical value), the crystallization is not stable (adapted from $[186,187])$.

[186] is given by

$$
\phi\left(u, X_{L}\right)_{\mathrm{rcp}, \mathrm{bim}}=0.64+0.184\left(1-X_{L}\right) X_{L}(u-1) .
$$

0.64 is the rcp for a a mono-sized system, $u$ is the diameter ratio of large and small spheres, $X_{L}$ the mole fraction of the large spheres.

Likewise, the packing fraction in crystalline phase for a bimodal distribution $\phi\left(u, X_{L}\right)_{\text {cryst,bim writes (for all the }}$ 14 Bravais lattices) [187]

$\phi\left(u, X_{L}\right)_{\text {cryst,bim }}=\frac{\eta_{\text {cryst }} X_{L}\left(u^{3}-1\right)+1}{X_{L}\left(u^{3}-1\right)+1+\left(1-X_{L}\right) X_{L}\left(u^{3}-1\right)}$,

where $\phi_{\text {cryst }}$ is the packing for a crystalline form ( 0.74 in fcc structure).

Figure 17 displays the phase diagram for a set of parameters, $X_{L}$ and $u$. Without interaction between particles, the stability is given by the maximum of compacity. In rcp the maximum of compacity is obtained for $X_{L}=X_{S}=0.5\left(X_{S}\right.$ is the mole fraction of small spheres). Below $u=1.2$, the stability of the crystalline phase (fcc in this example) is checked in the whole range of $X_{L}$. For $u>1.2$, the stability of the crystal phase is assumed outside the crossover labelled by A,B,C,D. For $X_{L}$ inside the range $\mathrm{AA}, \mathrm{BB}, \mathrm{CC}$ and $\mathrm{DD}$, the amorphous phase is expected to be the stable one. The main result is that the random bimodal distribution allows non-dense packing in a very narrow window. For fcc structure the critical value is about $u=1.2$ giving a packing fraction of about 0.66 compared to 0.74 in fcc structure. Hynninen et al. have numerically shown that both the pyrochlore and diamond structures could be obtained through selfassembly of a binary mixture of large and small colloidal spheres [188]. When the size ratio of the two populations of spheres is well defined, the so-called Laves phase

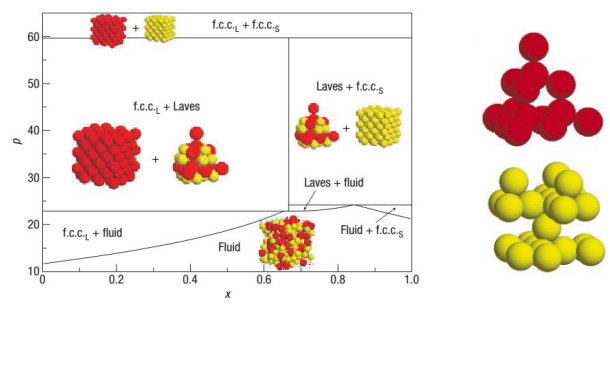

FIG. 18. Left. Phase diagram of binary hard spheres with a small-to-large size ratio of 0.82 . The phase diagram is shown in the composition $x$ (number fraction of small spheres) and reduced pressure $p$ representation. The labels f.c.c.L' and f.c.c.S' denote the fcc crystals of large and small particles, respectively. Right. Diamond structure of large spheres and pyrochlore structure of small spheres. Reprinted by permission from Macmillan Publishers Ltd: [188], copyright 2007.

$\mathrm{MgCu}_{2}$, into which the large spheres form two interpenetrating fcc lattices, whereas the small spheres sit at the vertices of a tetrahedron, is obtained (Fig. 18 left). Removing selectively the large or the small spheres from the $\mathrm{MgCu}_{2}$ phase leads to a pyrochlore structure of small spheres and a diamond structure of large spheres, respectively (Fig. 18 right).

\section{B. Mixture of isotropic/anisotropic particles}

Bimodal distributions of spherical particles and anisotropic polyhedral blocks can be assembled together. The lattice is given by the polyhedral block acting as a spacer [189]. Both types of particles are functionalized with DNA strands, allowing to encode particle interactions via DNA sequence.

The local and the superlattice structure (here the sphere packing) is given by the symmetry of the nanoblocks while interactions between particles are induced by functionalizing spheric NPs and cubic NPs with complementary DNA strands (Fig. 19). For that purpose, samples assembled at room temperature were subsequently annealed at a pre-melting temperature (234 K) for several hours, followed by gradually cooling back to room temperature. This ensures the crystallisation in the $\mathrm{NaCl}$ structure. Ducrot et al. [190] prepared preassembled colloidal tetrahedra and spheres (polystyrene particles) obtaining a class of colloidal superstructures, including cubic and tetragonal colloidal crystals. The superlattice is made up of two interpenetrating sublattices, one diamond, and the other pyrochlore. The spheres of 


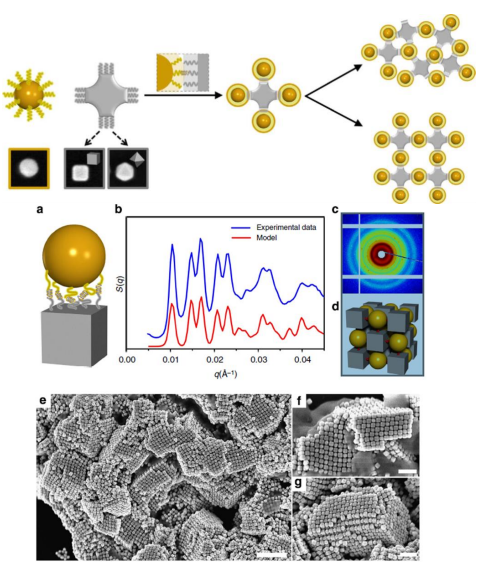

FIG. 19. Synopsis of the method. (a) Schematic of a $46 \mathrm{~nm}$ sphere/46 nm cubic NPs pair linked by intercomplementary DNA system. (b) Small-angle X-ray scattering (SAXS) data with experimental (blue) and modelled (red) structure factors, $S(q)$, (c) scattering image and (d) the corresponding structure schematic for $46 \mathrm{~nm}$ spherical/46 nm cubic NPs assembly system, which crystallizes into a NaCl-type lattice. (e) Low-magnification scanning electron microscope (SEM) image of shere/cubic-assembled crystals, where square-lattice ordering can be observed from the fragments. (f,g) Highmagnification images of superlattice, demonstrating the alternate packing of spheres and cubic NPs in the 3D square lattice (scale bar, 200nm) [189].

the diamond sublattice have four tetrahedrally coordinated nearest neighbours thanks to DNA-mediated interactions. The spheres of the pyrochlore sublattice can be grouped into distinct tetrahedral clusters that occupy the voids of the diamond sublattice.

\section{SUPRACRYSTAL BASED ON ANISOTROPIC FUNCTIONALIZED PARTICLES WITH INTERACTIONS}

As previously discussed, the assembly of particles interacting isotropically leads to close-packed lattices. In that sense, it allows to mimic metal crystals or ionic crystals if the colloidal precursors are pure batches of sizemonodisperse particles or binary mixtures of differentlysized particles [191], respectively. Nevertheless, less close-packed lattices such as covalent crystals remain unmimickable at the colloidal scale because their assembly necessitates highly directional interactions between building blocks. Indeed, the diamond structure, with a packing factor of 0.34 against 0.74 for fcc and hcp, is based on tetrahedral arrangements, i.e. fourfold coordination, of atoms displaying a fourfold valence, i.e. $s p^{3}$ orbital hybridization of carbon atoms. So, there is a huge interest in giving precursor colloids predetermined instructions for directional assembly by decorating their surface with sticky patches (enthalpic patches), or topological discontinuities such as dimples (entropic patches) for locking neighboring spherical colloids especially by depletion interactions. It shall be mentioned here that the self-assembly of tetravalent particles is not the unique route to get a diamond lattice of particles: some rare successful examples concerns the microfabrication technique [192] or caging of nanoparticles into tetrahedral DNA origamis [62] . These concepts of patchy particles and colloidal valence have been first implemented through digital experiments about one decade ago and led to a huge number of publications since. From the viewpoint of their effective fabrication, only few examples of patchy particles have been reported and their selfassembly capability has been actually checked to getting low-dimension systems such as discrete aggregates, linear chains or planar arrays. This led in particular to the ideas of colloidal molecules (CMs) and colloidal macromolecules (CMMs) opening the way to mimic at the colloidal scale any molecular entity existing at the atomic scale. It involves the development of robust and up scalable strategies to fabricating colloidal atoms (CAs) with valence of 1, 2, 3, 4 and even 6 to a certain extent. Monovalent, i.e. monopatchy, particles, usually called Janus particles [193], and their micelle-like or chain-like selfassemblies [193, 194] will not be described in this paper because they cannot lead to higher dimension lattices.

\section{A. Numerical self-assembly of patchy hard spheres}

Generally based on the Kern-Frenkel model [195], these digital experiments describe the phase behavior of square-well fluids with a patchy short-ranged attraction with the help of the conventional Monte Carlo simulation model. For obvious reasons of concision, only the studies concerning the assembly of pure batches of colloids with $2,3,4$ or 6 patches organized in linear, triangular, tetrahedral or octahedral arrangements, respectively, will be discussed below because they are the most representative of the fabrication efforts described in the next section. The assembly of divalent CAs with two identical patches has been extensively studied numerically. Sciortino and coworkers have developed a spot-like patch model to study a simple fluid composed of particles having a hardcore repulsion, complemented by two short-ranged attractive sticky spots at the particle poles [196]. They reported the formation of linear chains, which was in complete agreement with the predictions of the Wertheim theory [197]. The organization of two-patch spheres into chains whose average length increases smoothly with decreasing temperature was also observed by Glotzer and coworkers through a coarse-grained patchy model [198]. The patch-to-particle size ratio, i.e. the patch angular width, was particularly investigated and it was shown that, when this value is high enough to enable one patch to interact simultaneously with the patches of two neighboring particles, ring structures may be promoted leading to a two-dimensional Kagome lattice [199]. It has also been shown theoretically that triblock Janus parti- 
cles, which have two large attractive patches separated by a repulsive band, can form a 3D pyrochlore lattice [200]. Romano and Sciortino have further demonstrated by performing Monte Carlo simulations that a rational design of the shape and the symmetry of the two patches can drive the patchy particles to crystallize in a single morphology by eliminating the undesired polymorphs [201]. For instance, they have shown that triblock Janus particles patterned with staggered triangular patches, which is readily achievable within current synthesis methodologies [202], crystallize in $3 \mathrm{D}$ into a cubic tetrastack lattice. Some numerical studies have also concerned divalent CAs with two patches of different natures and/or sizes, especially the case of mono-dimple particles whose dimple radius is close to that of the particle. Ashton et al. have indeed studied their depletion-induced self-assembly and showed that they can assemble into chains in the presence of a depletant via lock-and-key binding in which the convex part of one particle interlocks with the concave part of another [203]. Sciortino and coworkers theoretically and numerically investigated the phase diagram of particles with three regularly arranged attractive patches [91, 92]. They mainly showed that the gas-liquid coexistence region in the temperature-density plane is reduced by comparison with particles with a higher number of patches. Particular attention has been paid to the specific case of tetravalent CAs with four identical patches arranged on a tetrahedral geometry because of their relevance to photonic crystals with diamond symmetry [92, 204-207]. Indeed, the phase diagram was found to be very rich, with several re-entrant coexistence lines and the range in density where the diamond phase is actually stable is very narrow, which is a consequence of its low compressibility. Moreover, at low pressures and finite temperatures, the diamond is competitive with a bcc crystal, both the diamond and the bcc exhibiting similar energies [208]. The diamond solid is only stabilized when the entropy increases, that is when the size of the patches decreases (Fig. 20). In fact, digital crystallization to the diamond structure is greatly favored when a seed crystal is introduced or when an additional term in the potential which induces a relative orientation between particles is added [94]. Glotzer and coworkers [209] showed how specific self-assembled structures can be targeted by engineering directional entropic forces through the systematic alteration of particle shape. They performed Monte Carlo simulations and demonstrated that tetrahedrally faceted spheres crystallize to a diamond lattice when the amount of facetting exceeds a certain value (Fig. 21a). One should note that the resulting diamond lattice exhibits a packing factor of at least 0.6 , which is noticeably higher than the one of the open diamond structure obtained from the self-assembly of particles with four enthalpic patches.

Several patchy particle models were considered to study the assembly behavior of six-patch octahedral particles [207, 210-212]. A bcc crystal appears more favorable at moderate pressures, whereas an orientationally
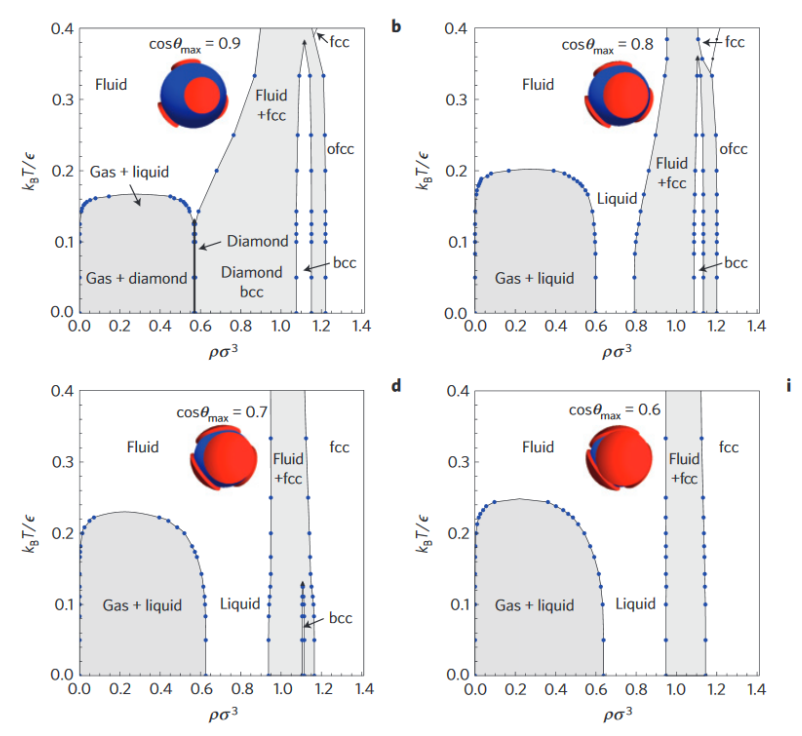

FIG. 20. Phase diagrams in the dimensionless densitytemperature representation for particles with four tetrahedrally arranged patches and different patch angular widths (patch width decreasing when $\cos _{\max }$ increases). Reprinted by permission from Macmillan Publishers Ltd: [208], copyright 2013.
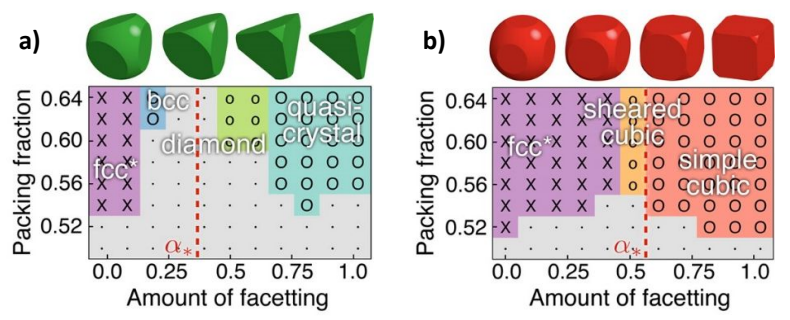

FIG. 21. Different crystal structures formed by assembly of a) tetrahedrally and b) cubically faceted spheres as the amount of faceting varies. Reprinted with permission from [209]. Copyright 2014 American Chemical Society.

ordered fcc structure becomes favorable at high pressures and low temperatures, and a fcc plastic crystal is the most stable solid phase at high temperatures. By slicing cubically coordinated facets into hard spheres, particles with six attractive entropic patches were created [209]. Monte Carlo simulations showed that such particles assemble into simple cubic lattices when the faceting amount and packing factors exceed 0.6 and 0.54 , respec- 
tively (Fig. 21b).

\section{B. Synthesis and self-assembly of particles bearing two and more patches}

As previously reported, anisotropic interactions may occur spontaneously between facetted particles. In addition, the differentially-reactive facets can be used for creating attractive patches quite easily. One of the most representative examples was reported by Kumacheva and coworkers who used hydrophobic interactions for binding metal nanorods by their ends [213]. They used for instance gold nanorods covered with a double layer of cetyl trimethylammonium bromide (CTAB) whose symmetry was broken by the selective exchange of the CTAB molecules anchored to the 111 facets (at the ends of the nanorods) by thiol-terminated polystyrene (PS) macromolecules. By selectively changing the quality of the solvent for the stabilizing molecules, i.e. PS and CTAB, they assembled the nanorods in rings, nanochains, bundles, nanospheres and bundled nanochains. They succeeded in getting CMMs mimicking copolymers by using a mixture of gold and palladium nanorods and controlling the length of the CMMs by using $\mathrm{Fe}_{3} \mathrm{O}_{4}$ nanoparticles bearing a gold patch serving as chain stoppers. The next examples concern amorphous and therefore spherical CAs whose symmetry is broken through different strategies to create surface patches or dimples. Original divalent CAs were obtained by Sacanna et al. from monodisperse silicon oil droplets nucleated from a homogeneous solution of hydrolyzed polymerizable alkoxysilane and encapsulated into cross-linked polymer shells [214]. The as-obtained microparticles bear one dimple whose diameter was close to that of the particles. In depletion condition they observed the formation of CMMs (lock-and-key strategy) similar to those obtained by simulation [203] and the absence of irreversible chemical bonds between the CAs allows these ball-in-socket joints to move freely. Using microparticles with a high patchto-particle size ratio, Granick and coworkers observed the two-dimensional Kagome lattice [215] envisioned digitally by Sciortino and coworkers [60]. They fabricated divalent particles made up of two hydrophobic patches separating an electrically charged band. These particles were fabricated by sequential deposition of metal on a monolayer of latex spheres. After the first vapor deposition, a polydimethylsiloxane (PDMS) stamp was used to lift up the monolayer so that patches are facing down. A second deposition is then performed to create patches on the other pole of the spheres and the grafting of n-octadecanethiol molecules onto the metal made the patches hydrophobic. As far as we know, no experimental study of the crystallization of trivalent or tetravalent CAs has been reported yet, even though several routes to produce such colloids exist. Indeed only discrete assemblies, i.e. CMs, were reported from CAs at microscale and therefore observed by optical or fluorescence mi- croscopies. It may be emphasized that linear, triangular, tetrahedral or octahedral geometries at the colloidal level have been systematically generated by steric hindrance, while at the atomic level they are caused by electron repulsion. The relevant examples from the literature consist in confining a controlled number of spheres (supposed to become the patches, patch precursors or dimple precursors) in a droplet, through sticky protrusions or at the surface of another colloid. Thus, mixtures of patchy microparticles were obtained by sonication of oil-in-water emulsions stabilized with negatively-charged PS particles and subsequent photopolymerization of the oil [216]. Other mixtures were produced through the assembly of polymer particles bearing a liquid protrusion and the subsequent polymerization of the liquid $[217,218]$. The direct confinement in droplets, initially developed by Lauga et al. [219], was largely exploited by Pine and coworkers [56, 220-223], in particular with $540 \mathrm{~nm}$ amidinated cross-linked PS spheres. By swelling the clusters with styrene, then polymerizing the styrene, islands of the original spheres protruding from the newly formed surface were functionalized with single-stranded DNA molecules. Their spontaneous assembly with other patchy particles bearing complementary DNA strands into AB-, AB2-, AB3-, and AB4-like CMs was observed by optical microscopy. Deriving this strategy with silica microspheres, they also fabricated multicavity particles and studied their three-dimensional assembly into CMs according to the lock-and-key route (Fig. 22).

Duguet, Ravaine and coworkers reported another fabrication route leading to silica nanoparticles with diameters of about $100 \mathrm{~nm}$ bearing patches whose number may be easily controlled from 1 to 12 with morphology yields reaching 70-80\% for particles with $1,2,3,4,6$ or 12 patches) [224-228]. They used a seeded-growth emulsion polymerization of styrene in the presence of silica particles previously surface-modified with methacryloxymethyltriethoxysilane (MMS), and obtained for instance silica/PS tetrapods made of a silica core and four tetrahedrally-arranged PS satellites (Fig. 23a). They showed it is possible to derive them into $\mathrm{sp}^{3}$-like dimpled CAs by simply dissolving the PS in THF (Fig. 23b) obtaining tetravalent particles whose shape is very close to that envisioned by Glotzer and coworkers (Fig. 21a). Taking advantage of the PS residue resulting from the copolymerization with MMS-derived grafts, the bottom of the dimples can be regioselectively functionalized, thereby leading to particles with potentially both enthalpic and entropic patchiness and therefore made capable to accommodate covalently-bonded spherical satellites (Fig. 23c). 


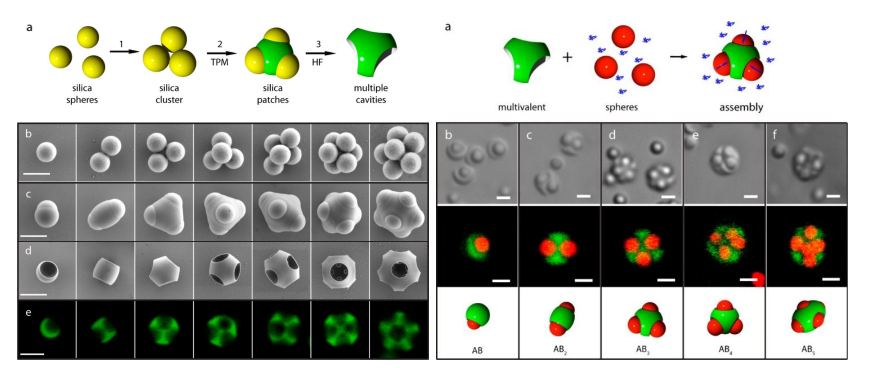

FIG. 22. Left. Multicavity micro particle fabrication. (a) Synthesis route: 1. A cluster of three silica spheres is prepared via an emulsionencapsulation process. 2. Partial encapsulation of the cluster with 3-(trimethoxysilyl)propylmethacrylate (TPM). 3. Silica clusters are etched out via treatment with hydrofluoric acid. Electron micrographs of (b) silica clusters, (c) silica-TPM patchy particles, and (d) multicavity particles. (e) Confocal fluorescent images of fluorescently labeled multicavity particles. Right. Three-dimensional lock-and-key assemblies. (a) Schematic illustration showing a trivalent particle with three assembled spheres using depletion interaction. The depletant (blue coil) causes osmotic pressure (arrows) between adjacent colloids, which is maximized when a sphere assembles into a cavity. (bf) Bright field micrographs (top panel), confocal micrographs (middle panel), and cartoons (bottom panel) showing multivalent lock particles with (b) one, (c) two, (d) three, (e) four, and (f) five cavities binding to red fluorescent spheres stoichiometrically. Scale bars: $1 \mu \mathrm{m}$. Reprinted with permission from [56]. Copyright 2014 American Chemical Society.

\section{DNA}

\section{NPs directionnaly functionalized with DNA}

The first achievements of the crystallization of nanoparticles into fcc and bcc superlattices driven by DNA assembly were only reported in 2008 [230, 231] followed by the achievement of binary lattices from gold nanoparticles and quantum dots or including a population of fake nanoparticles [232] (where the inorganic core has been dissolved to produce hollow DNA nanoparticles). Theoretical works predict that nanoparticles directionally functionalized by attaching different DNA strands at different locations can be designed to selfassemble into extremely complex architectures, such as close-packed crystals (tetrahedra, octahedra, icosahedra, etc.) [233] or the Empire State Building [234] (Fig. 24). However, such structures have not been achieved yet, by lack of appropriate building blocks, showing the importance of finding strategies to functionalize nanoparticles directionally.

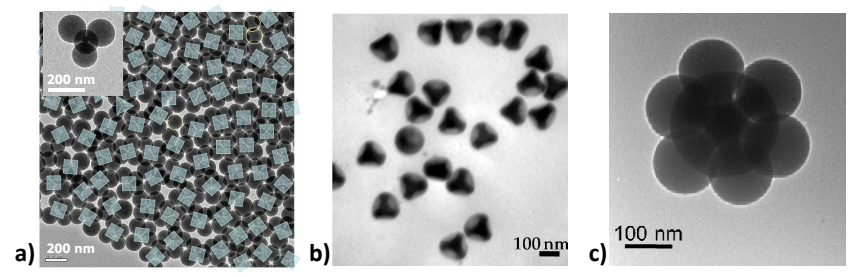

FIG. 23. a) Transmission electron microscopy (TEM) image of silica/PS tetrapods obtained by seeded-growth emulsion polymerization (each tetrapod has been labelled in blue and 3 free PS latex particles in yellow; scale bar: $200 \mathrm{~nm}$ ) (Reproduced from [225] with permission of The Royal Society of Chemistry). b) TEM image of derived $\mathrm{Csp}^{3}$-like silica particles with four dimples, i.e. entropic patches. (Reprinted with permission from [226]. c) TEM image showing how six silica spheres may be securely locked into the six dimples of silica particles (deriving here from silica/PS hexapods) through peptidic bonding, i.e. aminated PS at the bottom of the dimples and activated carboxylic groups on the silica satellites [229].

As a result of shape complementarity, 1D chains of alternating cubes and disks were formed by DNA mediated co-cristallization, when the diameter of the disk is larger than the length of the cube [4, 235] (Fig. 25).

\section{Origamis}

DNA folding can be programmed to produce 2D and 3D architectures (figure 26), including lines, cubes, and smileys, etc. referred to as origami. DNA origami are fully addressable, enabling further functionalization in a programmable manner and numerical studies to explain how such a robust self-assembly occurs have been proposed recently by Frenkel et al. [128, 236-238]. The positioning of nanoparticles onto DNA origamis $1 \mathrm{D}, 2 \mathrm{D}$ or $3 \mathrm{D}$ templates with a precision of the order of the nanometer was successfully achieved, with high yields. Thus, rationally designed plasmonic materials such as chiral helices based on metallic particles grafted onto a DNA scaffold were demonstrated [239]. DNA programmed folding can also form monodisperse 3D origami nano-objects which can be turned into versatile patchy building blocks for anisotropic assembly. Thus, the assembly of DNA cuboids via lateral faces yields $1 \mathrm{D}$ chains [240]. The key point of this strategy is to decouple the formation of the cuboids from their assembly, by selecting DNA connector sequences of very different melting temperature. 


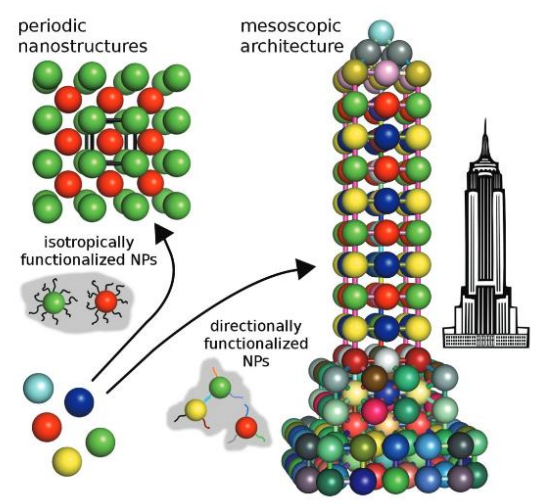

FIG. 24. Nanoparticles isotropically functionalized with complementary single-stranded DNA self-assemble into periodic nanostructures like a body centered cubic crystal. When the nanoparticles are directionally functionalized by attaching DNA strands at specific locations, the particles can be designed to self assemble into finite-size mesoscopic architectures, such as the Empire State Building. From [234].
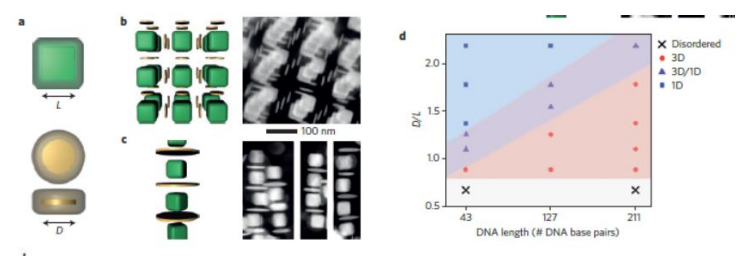

FIG. 25. Programming colloidal crystal habit with anisotropic nanoparticle building blocks and DNA bonds. From [4].

\section{DNA transplant onto NPs with patchs}

The symmetry of surface functionalization can be broken by manipulating the core/shell structure of polymer coated NPs. Thus, the competition between a hydrophilic and a hydrophobic low molecular weight thiolated ligand at the surface of gold NPs, regulates the partial attachment of an amphiphilic PS-b-PAA polymer [242]). When only hydrophobic ligand is present, a homocentric core/shell (Au@PS-PAA) structure is formed whereas in the presence of a mixture of hydrophilic and hydrophobic ligand, an eccentric core-shell is formed, exposing partially the surface of the particle to the outer media (Fig. 27). DNA grafted to this exposed surface further allowed the elaboration of dimers, tetramers, "cat-
(A)

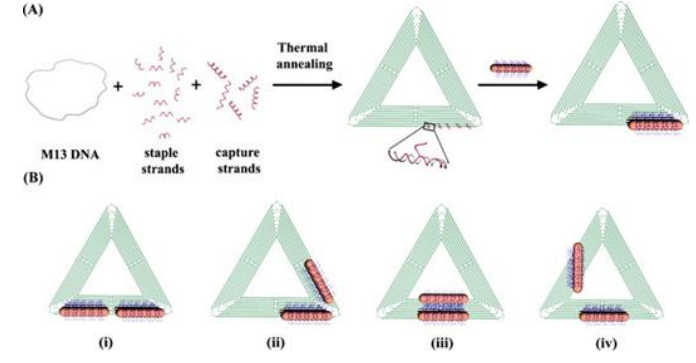

FIG. 26. DNA programmed folding can also form monodisperse 3D origami nano-objects which can be turned into versatile patchy building blocks for anisotropic assembly. Thus, the assembly of DNA cuboids via lateral faces yields 1D chains [240]. The key point of this strategy is to decouple the formation of the cuboids from their assembly, by selecting DNA connector sequences of very different melting temperature. From [241].

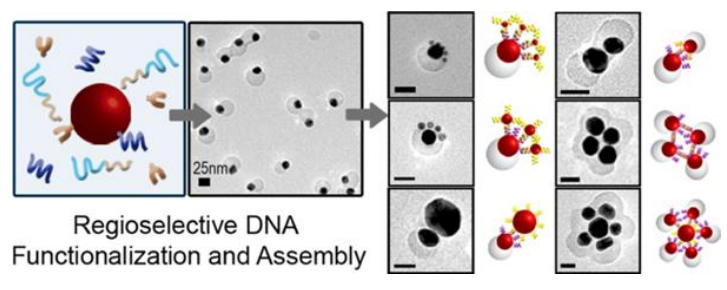

FIG. 27. Cat paws, dimers and tetramers formed from DNA functionalized eccentric Au/PS-PAA core-shell nanoparticles. From [243].

paw" and satellite flower assemblies, with high regioselectivity and monodispersity.

\section{X. (EXPECTED) PROPERTIES OF ANISOTROPIC SELF-ASSEMBLIES OF NPS AND POSSIBLE APPLICATIONS}

The self-assembly of anisotropic particles has gained a great interest recently, and significant advances have to be pointed out $[87,215,244,245]$ opening the door to application developments. 


\section{A. Optoelectronics: photonic crystals}

Photonic crystals are $1 \mathrm{D}, 2 \mathrm{D}$ or $3 \mathrm{D}$ periodic structures constructed from alternating regions with different refractive indices that promote a photonic band gap (PBG), analogous to the electronic band gap, which facilitates control of electrons in atomic crystals [246-250]. The position of the bandgap can be shifted by either changing the properties of the two constitutive materials and/or by changing the lattice constant, while the width of the bandgap can be widened by increasing the difference between the dielectric constant of both materials. As a result, photonic crystals have been proposed for use as a powerful means to manipulate, confine, and control light in three dimensions of space. The fabrication of $3 \mathrm{D}$ photonic crystals exhibiting a PBG in the optical regime in large quantities, at a reasonable cost, and in a controllable way, is still a great challenge for materials scientists. In order to get a bandgap in the visible region, one needs to pattern the dielectric materials into structures of 100-300 nm in size along all three dimensions. Even if such feature sizes can be obtained with top-down approaches such as three-dimensional holographic interference lithography and two-photon lithography, these techniques are generally expensive and require a longer time to fabricate the structures over a larger area because of the serial nature of the approach. Colloid based solutions are highly attractive as an alternative method due to the ease of accessing the submicrometer rsize regime. These bottom-up approaches rely on the self-driven assembly of pre-designed spherical (or, more rarely, anisotropic) building blocks into ordered periodic structures which are close to or at a thermodynamic equilibrium state. The cost-effective production of 3D photonic structures (mostly fcc lattices) with a bandgap in the visible region through colloidal self-assembly has indeed been extensively reported [251-253].

The real push in this field has been the strong desire to achieve the fabrication of $3 \mathrm{D}$ photonic crystals possessing a complete photonic band gap (PBG) at optical wavelengths. A complete $\mathrm{PBG}$ is a range of frequencies for which light propagation is forbidden regardless of propagation direction. 3D photonic crystals with a complete PBG offer a vast number of applications such as low-loss waveguides, platforms for optical microcircuits, and thresholdless lasers [249, 254, 255]. Many computational studies have pointed out the conditions (e.g., the symmetry of the lattice points, the structural type of the lattice, and the minimum contrast in refractive index between high and low dielectric regions) under which photonic crystals should exhibit complete bandgaps in the optical regime [256-260]. Unfortunately, it was demonstrated that a fcc structure, that is mostly obtained by self-assembly of colloidal spheres, is not well-suited to generate photonic crystals with a complete bandgap due to degeneracy in the photonic band structure caused by the spherical symmetry of the lattice points [261, 262]. In order to lift degeneracy at points of high symmetry in the Brillouin zone, and thus to obtain a complete PBG, it was suggested to employ non-spherical objects as the building blocks to construct fcc lattices via self-assembly. The results indicated that a complete bandgap could develop between the second and third bands in the photonic structure as dimer-like particles consisting of two interconnected spheres with identical [261, 263] or different radii $[264,265]$ were used as building blocks. One should note that such dimers can be easily synthesized by phase separation in a seeded-polymerization technique [266] or by seeded emulsion polymerization [267]. The self-assembly of PS dimer- and spherocylinder-shaped colloids was recently achieved by evaporation mediated vertical deposition but only a partial photonic bandgap was experimentally observed for crystals from spherocylinder building blocks [268]. Computational studies have also suggested another strategy to obtain a complete PBG, by reducing the symmetry of the 3D lattice from the fcc to diamond or pyrochlore (also known as tetrastack) structures. In these cases, a complete gap was shown to develop for a minimum refractive-index contrast ratio of 1.9 and at low-lying bands [269-272], which makes the structures more stable against disorder [273]. Although the diamond and pyrochlore structures are very well suited to fabricate crystals with a complete PBG, their formation is not favoured by spontaneous colloidal self-assembly due to their low packing densities. In order to capitalize on the advantage of colloids for lowcost fabrication, several methods have been proposed to overcome this limitation. Ngo et al. have suggested the use of four spheres combined in the form of tetrahedrons as building units to form a tetrastack structure [274].

As mentioned in Sec. VIII pyrochlore and diamond structures could be obtained through self-assembly of a binary mixture of large and small colloidal spheres. However, most promising route to fabricate diamond or pyrochlore structures by self-assembly consists in decorating the surface of colloids with attractive patches [275]. Because of the tetrahedral packing of the diamond and pyrochlore lattices, particular attention has been paid to the specific case of four identical patches arranged on a tetrahedral geometry [204, 276, 277]. Nevertheless, obtaining a diamond-like or pyrochlore-like lattice will not be necessarily sufficient to make the colloidal crystal working as a complete PBG. It will be indeed mandatory to adjust the refractive-index contrast ratio between the colloids and the voids between the colloids at values higher than 1.9. If the precursor colloids cannot be high index materials, e.g. titania, filling the interparticular voids with a high index material and subsequently dissolving the colloids to get an inverse crystal will be an alternative.

\section{B. Metamaterials}

Metamaterials are composite systems whose properties are dominated not by the individual atoms, but by 


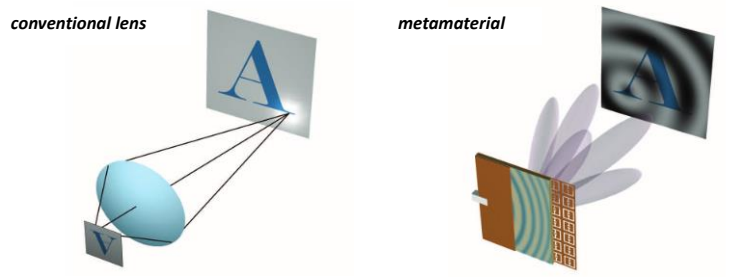

FIG. 28. The imaging device consists of a leaky waveguide, formed by patterning the top conductor of a standard microstrip line with complementary electric-inductor-capacitors (cELCs). The planar waveguide feeds a holographic array of ELCs, removing the need for lenses. The waveguide acts as a coherent single-pixel device, with the array of ELCs serving to produce the illuminating complex spatial modes after the original figure from [279]. Conventional lenses only capture two dimensions of a three-dimensional object, flat lens is able to project three-dimensional images of three-dimensional objects.

the properties of larger, artificially produced structures or meta-atoms. They are engineered structures designed to interact with electromagnetic radiation in a desired fashion (negative index). Smith et al. first demonstrated [278] that a supracrystal based on a periodic array of interspaced conducting nonmagnetic split ring resonators and continuous wires exhibits a frequency region in the microwave regime with simultaneously negative values of effective permeability and permittivity. Among the properties, the most amazing is the negative index metamaterial. All materials have an index of refraction, which measures the degree and direction that light is bent as it passes through these materials. This index of refraction is basically an intrinsic property of the matter. One of the application is the design of flat lens [279] that avoids aberrations that plague glass lenses with positive refraction index have (Fig. 28). Another is the superlens. Diffraction limit dictates that no conventional lens (positive index) can resolve details much smaller than the wavelength of the light that illuminates its target. Flat lenses could surpass such limits.

\section{Plasmonics}

Plasmonics in superlattices has been extensively studied over the two past decades [280-282]. Plasmon band in a metallic cluster with a size lower than the light wavelength obeys to the Mie dipole response. The optical properties of a supra (metallic)lattice can be considered as a collection of interacting dipoles. If the superlattice
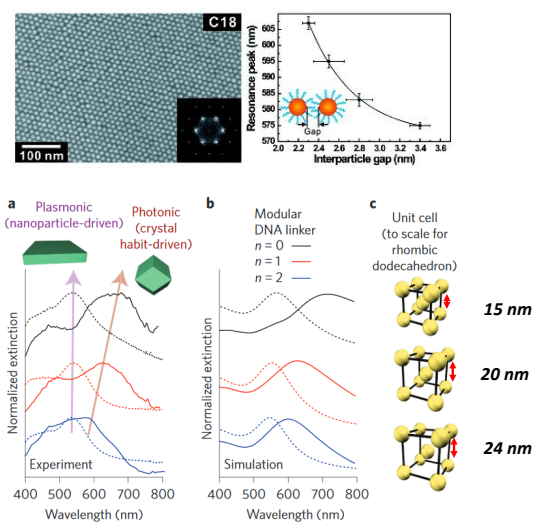

FIG. 29. Top: (dense hcp packing) Alkanethiolate-stabilized gold nanoparticle superlattices observed by SEM and the relation between interparticle gap and the plasmon peak, the gold cluster has a mean size of $20 \mathrm{~nm}$. The interparticle gap is fixed by the alkyl chains (after [281]). Bottom: (cc packing) comparison of the plasmon frequency shift for a thin (superlattice) film of $150 \mathrm{~nm}\left(1 \mathrm{~cm}^{2}\right.$ square) and rhombic dodecahedra crystals $(1 \mu \mathrm{m})$. The shift is 15 and $100 \mathrm{~nm}$ respectively for thin film and dodecahedra (after [283]).

is well ordered the dipoles can interact with a collective response [281]. The shape of the superlattice plays an important role when the size is of the same order of magnitude than the light wavelength. Ross et al. [283] prepared DNA-programmable assembly of mesoscale superlattices of $20 \mathrm{~nm}$ gold spheres. The lattice parameter is fixed by the DNA design which constitutes the spacer. Figure 29 shows the tuning of the plasmon band according to the lattice parameter for a macroscopic sample and small rhombic dodecahedra $(1 \mu \mathrm{m})$. The plasmon shift is enhanced in this case and can be attributed to a photonic crystal effet.

\section{Application in heterogeneous catalysis}

2D-assembly of metallic nanoparticles has already been used as model catalysts especially to study the effects of size, shape and density of particles on the catalytic properties. This work has been recently reviewed [285]. Here, we are focused on 3D assembly of particles. This kind of assembly is until now much less used for catalytic application but it presents interesting perspectives that we will shortly described in this paragraph. 3D-assembly of colloids is used to form porous membranes which can be used as catalysts. These membranes are made in several steps. First, preparing a regular dense assembly of polymer or silica spherical particles (the same method could be also used with non-isotropic assembly), then precursors in solution are introduced in the free space between the colloids, then by calcination the precursors form a solid framework. Latter on, the template particles are eliminated by calcination at high temperature or 


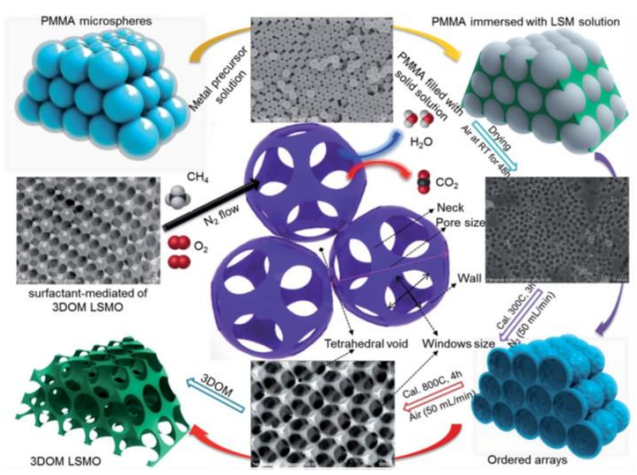

FIG. 30. The different fabrication stages of a 3DOM $\mathrm{La}_{0.6} \mathrm{Sr}_{0.4} \mathrm{MnO}_{3}$ (LSMO) catalytic membrane prepared from a PMMA microspheres assembly. From [284]

by chemical etching. After removing the colloid particles template, the solid porous membrane is formed. If it is disordered it is called colloid-based porous material (CBPM), if it is ordered it is called three-dimensional ordered macroporous (3DOM) membrane or inverse colloidal crystal [286] . The material from the membrane can be a metal oxide with catalytic properties $\left(\mathrm{TiO}_{2}\right.$, $\mathrm{CeO}_{2}, \mathrm{MnO}_{2}$ ) or a complex oxide with a perovskite or a spinel structure (see Fig. 30) [284]. The 3DOM membranes present generally a better catalytic activity than the powder of the same oxide, moreover the presence of large pores (typically $100 \mathrm{~nm}$ ) facilitates the mass transfer. In the case of reactions which need a transition metal, catalyst nanoparticles ( $\mathrm{Au}, \mathrm{Ag}, \mathrm{Pt}, \mathrm{Pd}, \mathrm{AuPd}$, etc) uniformly distributed on the inside walls of the membrane are obtained by adding a metallic salt during the synthesis of the porous membrane [287-289]. A very promising application of $3 \mathrm{DOM} \mathrm{TiO} 2$ with $\mathrm{Au}$ nanoparticles is photocatalysis [290, 291]. Indeed very high activity (larger than $\mathrm{Au} / \mathrm{TiO}_{2}$ powder catalysts) has been obtained for the reduction of $\mathrm{CO}_{2}$ in methane with visible light. This high performance is due to two factors: large photon harvesting due to the photonic properties of the $3 \mathrm{DOM}$ structure (like in the case of a photonic crystal), plasmon resonance excitation of hot electron in the gold nanoparticles which are injected in the conduction band of the $\mathrm{TiO}_{2}$ which then activate the $\mathrm{CO}_{2}$ molecule.

Assembly of colloidal metallic particles can be used in heterogeneous catalysis but two problems arise. First, the stabilizing ligands around the particles limit the number of free metal sites. Second, the assembly is not stable during catalysis because some coalescence occurs between the metallic particles which is due to the partial desorption of the ligands. An alternative solution has been recently put forward by Bäumer et al. [292]. It is based on the use of bifunctional ligands which link two particles by their two end groups. By using ligands formed by an alkyl chain with two terminal amine groups which bound to Pt nanoparticles, a solid porous material is obtained. In this material $50 \%$ of the surface atoms of the particle remain free and the catalytic activity presents a good stability [292]. This new method has been used for hydrogen gas sensing application [292]. If these new concepts of catalytic membranes present very promising properties they are not yet employed in industrial catalysis. This is due to two main reasons (i) the fabrication cost of $3 \mathrm{DOM}$ membranes is very high compared to the classical impregnation method used for the fabrication of industrial catalysts, (ii) the size of the membranes obtained until now is too small for industrial application. However, these two limitations could be overpassed by some further developments in particular in the appealing fields of photocatalysis or electrocatalysis [293].

\section{E. Magnetism}

NPs exhibit superparamagnetism when magnetization can randomly flip direction under the influence of temperature. Blockage appears when the volume is high enough (the anisotropy energy is proportional to the volume) to counterbalance the thermal energy. Superparamagnetism can be bypassed when NPs are coupled together in a network. Then, the blocking temperature increases due to the collective behavior. In this case, the mean NPs distance and the neighboring are the main parameters whatever the long range ordering. Pileni et al. [294] showed that the nanocrystals ordering on long distance in supracrystals permits the emergence of collective intrinsic properties (Fig. 31). Generally, the NP acts as a magnetic monodomain with a giant magnetic moment (superspin which is a zero dimensional magnetic building block) [295]. The open issue is the complexity of interparticle interactions, the standad one being the magnetic dipolar forces [296-301]. Since the geometry of the supracrystal can be controlled, it is possible to create geometric frustration with no degeneracy (for example in triangular lattice) or degeneracy in Kagome lattice. However, also direct and superexchange mechanisms may exert an influence in the long range ordering.

Superparamagnetic colloidal photonic crystals can be built. Adding magnetic components to the colloidal building blocks provides an opportunity for convenient and precise control of the properties of photonic crystals through an external magnetic field [302] (Fig. 32).

\section{F. Energy storage}

Supracrystal can be used as a template to build up ordered mesoporous structures. (Fig. 33). Guo et al. [303] shown that ruthenium decorated hierachically ordered mesoporous carbon can be used as stable electrodes for lithium oxygen batteries. 

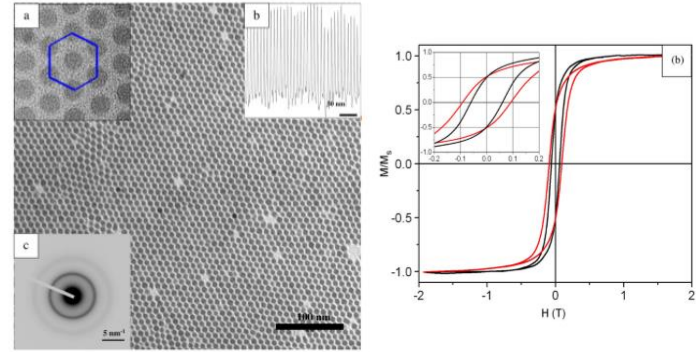

FIG. 31. (a) TEM image of a hexagonal network of cobalt NPS (left) and (right) magnetization curves of disordered network (black) and ordered hexagonal network (red) [294]. The hysteresis opening in ordered network is due to additional coupling at long range order.

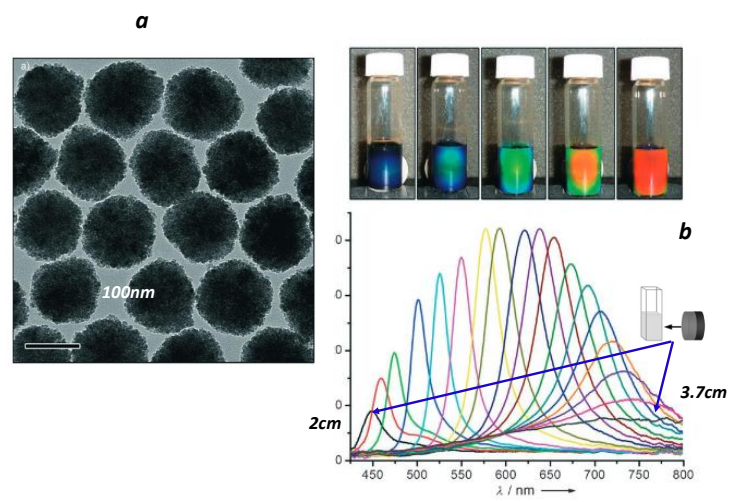

FIG. 32. (a) TEM image of polyacrylate capped $\mathrm{Fe}_{3} \mathrm{O}_{4}$ colloidal NPs. The magnetic particles are in the superparamagnetism regime. The reflection spectra at normal incidence can be tuned applying a magnetic field, tuning parameter is the distance between the magnet and the sample (each curve is separated by a step of $0.1 \mathrm{~cm}$ ) [302].

\section{G. Mechanical properties of TPMS supracrystal}

\section{1. outlook}

From a mechanical point of view, the supracrystal can be looked at a porous solid [304]. Among the huge zoology of porous structures, the mechanical properties of porous solids based on the labyrinth domains of triplyperiodic minimal surfaces S (TPMS) are renewed a considerable interest over the past decade with the development of the additive manufacturing processes. TPMS surfaces have two important properties: the surfaces locally minimize their areas which is equivalent to having zero mean curvature and they have the symmetries of a

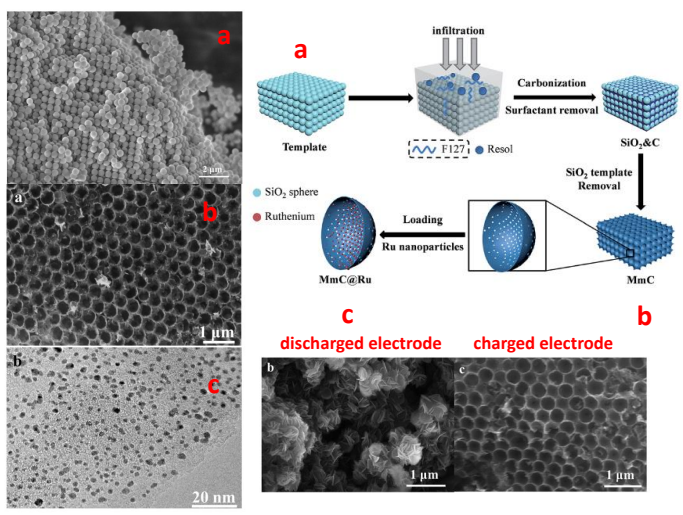

FIG. 33. (a) Synopsis of the electrode engineering. SEM image of the $\mathrm{SiO}_{2}$ template (a), the carbon mesoporous structure (b) and the ruthenium clusters (TEM observation) encaged in the mesopore (c). The morphology of the electrode after charge and discharge is shown. After the original figures in Ref. [303].
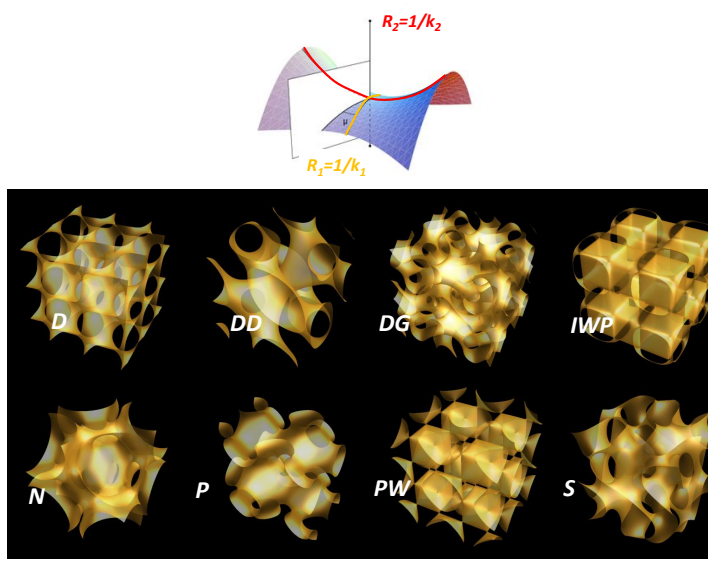

FIG. 34. Definition of the curvature parameters in minimal surface. On a minimal surface the curvature along the principal curvature planes are equal and opposite at every point. Few examples of TPMS are displayed at the bottom. The TMPS discussed in the text corresponds to the labeled "P".

crystallographic group in $\mathbb{R}^{3}$. The surfaces (locally) are characterized by a set of two curvatures: the mean curvature $\left(k_{1}+k_{2}\right) / 2$ and the Gauss curvature $k_{1} k_{2}$. Taking as reference a flat surface $\left(k_{1}=k_{2}=0\right)$, a local nonzero curvature induces a stress according to the Willmore functional $\tilde{W}[305,306]$ (a more accurate form is given by the Helfrich energy [307] in physics but the idea is the same).

$$
\tilde{W}=\int_{S}\left\{\left[\left(k_{1}+k_{2}\right) / 2\right]^{2}-k_{1} k_{2}\right\} d S .
$$

The Willmore functional is invariant under conformal changes of metric (in other words is independent of the nature of the elements). This formalism can be used to qualitatively explain the cohesive energy as a function of 


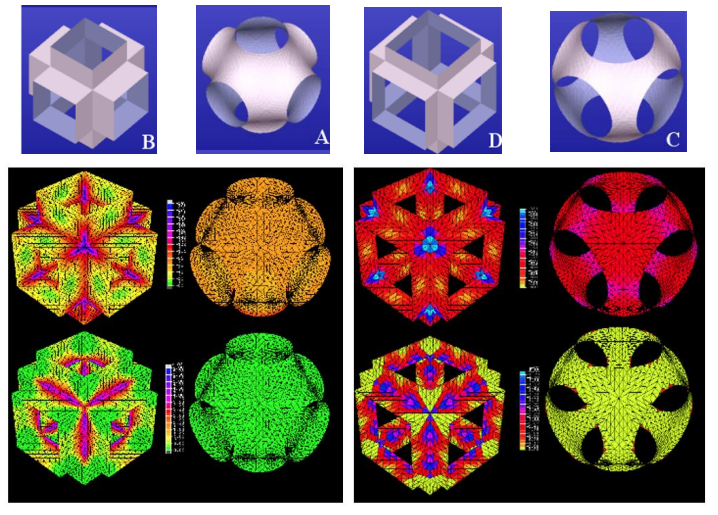

FIG. 35. left panel: Von Mises stress (top) and principal strain (bottom) maps under bulk compression for cubic (left) and TPMS (right) unit cells (scale factor 1.0) with identical loading conditions and material properties . Right panel: with a scale factor of 1.25. The corresponding TPMS surfaces are given at the top of the figures. Note the similarities between the TPMS with a scale of factor 1.25 and Fig. 30.

the curvature (radius) in fullerenes and single-wall carbon nanotubes (SWNTs) [308]. Note that in SWNTS the Gauss curvature is zero, in fullerenes the Gauss and mean curvatures are non zero and in TPMS the mean curvature is zero.

\section{Mechanical properties}

Mechanical properties of TPMS have been progressed over the past decade [309-313]. Among them, Von Mises stress is a tool for engineers giving an information if the design will fail under a load. Figure 35 displays the Von Mises stress and the principal strain in TPMS structures compared to classical superlattices with the same whole symmetry (cubic). We clearly see that, contrary to common structures, the stress/strain is homogeneously distributed over the network in the TPMS structures.

\section{H. The future of additive manufacturing}

Additive manufacturing [314], i.e. 3D printing, refers to fabrication processes used to synthesize a threedimensional object in which successive layers of material are formed under computer control to create an object. Early equipment and materials were developed in the 80 's and generally used for rapid prototyping. It is often claimed that it signals the beginning of a third industrial revolution. Today, several techniques coexist as a function of the chemical composition of the precursor powders: selective laser melting, selective laser sintering, electron beam melting, etc. Colloidal self-assembly could embody the future of these techniques, where the printer will be gone and it is matter, e.g. the building blocks, that will be programmed to spontaneously form in liquid media the desired objects and devices. Moreover, the consolidating stage currently achieved with a laser or electron beam would become useless, or replaced by an ultimate and global sintering stage. Colloidal selfassembly would allow to fabricate more easily multiphasic materials, contrarily to current techniques which use powders of identical chemical composition. Nevertheless, making this future technology successful involves programming the building blocks to specify not only the location and connectivity of the building blocks within the assembled structure, but probably also the order and manner in which they are added to it $[315,316]$. This encoded information determines the complexity of the final structure which at equilibrium minimizes the system's free energy and is determined by the accessible configurations and their respective energies. As discussed earlier, despite progress towards programmability, the selfassembly of colloidal materials remains limited to highly symmetric structures, e.g. periodic close-packed colloidal crystals, linear chains and small clusters, and cannot provide arbitrary structures. In the puzzle approach described by Cademartiri and Bishop, highly selective and directional interactions between colloids are necessary to lower the energy of the desired structure [316]. The number of specific interactions is much larger for arbitrary structures than for periodic structures, which are fully specified by their unit cell. Nevertheless, fabricating a device in solution means being capable to program the shape and the dimension of the assembled structures, i.e. deciding when the bulk structure ends and therefore when it is timely to place surface building blocks. For example, the assembly of colloids into a bcc crystal requires only one specific interaction, however programming a similar crystal of precisely 1,000 colloids and arbitrary shape would require 1,000 specific interactions. As far as we know, DNA is the only practical chemical system that can produce such large numbers of distinct and specific interactions whose strength can additionally be tuned by varying the number of bases in the sticky ends of the DNA strands [317, 318]. Encoding specificity may also be achieved in a much lesser extent by interactions based on complementary shapes. But to get arbitrary structures, the puzzle approach requires selective interactions that are both directional and independently addressable, i.e. arising from anisotropic and/or patchy chiral colloids. Although the puzzle approach simplifies the design of equilibrium structures, it offers little in the way of controlling the kinetic process of equilibration [316]. The lack of long-range correlations between the assembling components leads to flat energy landscapes, i.e. to many configurations of similar energy, which can result in very long assembly times and high probability of forming kinetically trapped structures. Annealing remains the simplest way to achieving the equilibrium structure. An alternative route would be tailoring the relative strengths of specific interactions for instance by 
determining the primary nucleation event.

\section{CONCLUSION/PERSPECTIVES}

"Large scale" synthetic of nanoscale organized materials constitutes a challenge for the community of chemists. Excepted promising toolbox based from non-additive forces derived from Casimir effect, most of the isotropic interactions between colloidal atoms favor compact structures. The explanation comes from the robustness of the universal scaling of the transition phases leading to dense packing. Supracrystals based on anisotropic particles with interactions seems a promising way for non-dense packing. The anisotropy can be monitored directly by the anisotropy of the surface in complex polyhedra or artificially by functionalized surfaces with ligands. Among them, NPs directionnaly functionalized with DNA is the best way for the future. It is a real challenge to overcome with the help of modeling/numerical predictions that must focus on unrealistic (unperfect) systems: the synergy between experimental and numerical research is mandatory.
[1] M. Lewenstein, A. Sanpera, V. Ahufinger, B. Damski, A. Sen, and U. Sen, Advances in Physics 56, 243 (2007).

[2] I. Bloch, J. Dalibard, and W. Zwerger, Reviews of Modern Physics 80, 885 (2008).

[3] G. V. Hartland, The Journal of Physical Chemistry Letters 2, 1111 (2011).

[4] M. N. O’ Brien, M. R. Jones, B. Lee, and C. A. Mirkin, Nature Materials 14, 833 (2015).

[5] R. L. Marson, T. D. Nguyen, and S. C. Glotzer, MRS Communications 5, 397 (2015).

[6] S. F. Tan, S. W. Chee, G. Lin, and U. Mirsaidov, Accounts of Chemical Research 50, 1303 (2017).

[7] R. L. Whetten, M. N. Shafigullin, J. T. Khoury, T. G. Schaaff, I. Vezmar, M. M. Alvarez, and A. Wilkinson, Accounts of Chemical Research 32, 397 (1999).

[8] M. Brust, D. J. Schiffrin, D. Bethell, and C. J. Kiely, Advanced Materials 7, 795 (1995).

[9] D. Grasso, K. Subramaniam, M. Butkus, K. Strevett, and J. Bergendahl, Reviews in Environmental Science and Biotechnology 1, 17 (2002).

[10] S. K. Ghosh and T. Pal, Chemical Reviews 107, 4797 (2007).

[11] J. J. Storhoff, A. A. Lazarides, R. C. Mucic, C. A. Mirkin, R. L. Letsinger, and G. C. Schatz, Journal of the American Chemical Society 122, 4640 (2000).

[12] N. Goubet and M.-P. Pileni, The Journal of Physical Chemistry Letters 2, 1024 (2011).

[13] M.-P. Pileni, Journal of Physics: Condensed Matter 23, 503102 (2011).

[14] W. I. F. David, R. M. Ibberson, J. C. Matthewman, K. Prassides, T. J. S. Dennis, J. P. Hare, H. W. Kroto, R. Taylor, and D. R. M. Walton, Nature 353, 147 (1991).

[15] J. Echeverría, D. Casanova, M. Llunell, P. Alemany, and S. Alvarez, Chemical Communications , 2717 (2008).

[16] M. A. Strzhemechny and E. A. Katz, Fullerenes, Nanotubes and Carbon Nanostructures 12, 281 (2005).

[17] E. A. Katz, D. Faiman, K. Iakoubovskii, A. Isakina, K. A. Yagotintsev, M. A. Strzhemechny, and I. Balberg, Journal of Applied Physics 93, 3401 (2003).

[18] Z. Zhong, S. Patskovskyy, P. Bouvrette, J. H. T. Luong, and A. Gedanken, The Journal of Physical Chemistry B 108, 4046 (2004).

[19] X. Ye, J. Chen, B. T. Diroll, and C. B. Murray, Nano Letters 13, 1291 (2013).
[20] S. A. Krasnikov, S. I. Bozhko, K. Radican, O. Lübben, B. E. Murphy, S.-R. Vadapoo, H.-C. Wu, M. Abid, V. N. Semenov, and I. V. Shvets, Nano Research 4, 194 (2011).

[21] Y. Ma, M. Eremets, A. R. Oganov, Y. Xie, I. Trojan, S. Medvedev, A. O. Lyakhov, M. Valle, and V. Prakapenka, Nature 458, 182 (2009).

[22] J. Hubbard, in Proceedings of the Royal Society of London A: Mathematical, Physical and Engineering Sciences, Vol. 276 (The Royal Society, 1963) pp. 238-257.

[23] G. M. Whitesides and B. Grzybowski, Science 295, 2418 (2002).

[24] S. C. Glotzer, M. J. Solomon, and N. A. Kotov, AIChE Journal 50, 2978 (2004).

[25] X. Bouju, C. Mattioli, G. Franc, A. Pujol, and A. Gourdon, Chemical Reviews 117, 1407 (2017).

[26] Y. Min, M. Akbulut, K. Kristiansen, Y. Golan, and J. Israelachvili, Nature Materials 7, 527 (2008).

[27] K. J. M. Bishop, C. E. Wilmer, S. Soh, and B. A. Grzybowski, Small 5, 1600 (2009).

[28] D. Luo, C. Yan, and T. Wang, Small 11, 5984 (2015).

[29] J. N. Israelachvili, Intermolecular and Surface Forces (Academic Press Inc. (London), 2011).

[30] D. V. Talapin, E. V. Shevchenko, C. B. Murray, A. V. Titov, and P. Král, Nano Letters 7, 1213 (2007).

[31] S.-W. Lee and W. M. Sigmund, Colloids and Surfaces A: Physicochemical and Engineering Aspects 204, 43 (2002).

[32] V. A. Parsegian, van der Waals Forces. A Handbook for Biologists, Chemists, Engineers, and Physicists (Cambridge University Press (Cambridge), 2006).

[33] I. E. Dzyaloshinskii, E. M. Lifshitz, and L. P. Pitaevskii, Physics-Uspekhi 4, 153 (1961).

[34] C. Girard and X. Bouju, Journal of Chemical Physics 95, 2056 (1991).

[35] C. Girard, X. Bouju, and C. Joachim, Chemical Physics 168, 203 (1992).

[36] H.-Y. Kim, J. O. Sofo, D. Velegol, M. W. Cole, and A. A. Lucas, Langmuir 23, 1735 (2007).

[37] B. V. Derjaguin and L. Landau, Acta Physicochimica USSR 14, 633 (1941).

[38] E. J. W. Verwey, The Journal of Physical and Colloid Chemistry 51, 631 (1947).

[39] E. J. W. Verwey and J. T. G. Overbeek, Theory of the stability of lyophobic colloids (Dover Publications (Mineola, N. Y.), 1999). 
[40] E. V. Shevchenko, D. V. Talapin, N. A. Kotov, S. O’ Brien, and C. B. Murray, Nature 439, 55 (2006).

[41] A. M. Kalsin, M. Fialkowski, M. Paszewski, S. K. Smoukov, K. J. M. Bishop, and B. A. Grzybowski, Science 312, 420 (2006).

[42] V. Liljeström, J. Seitsonen, and M. A. Kostiainen, ACS Nano 9, 11278 (2015).

[43] J. Faraudo, J. S. Andreu, C. Calero, and J. Camacho, Advanced Functional Materials 26, 3837 (2016).

[44] J. Hernández-Rojas, D. Chakrabarti, and D. J. Wales, Physical Chemistry Chemical Physics 18, 26579 (2016).

[45] B. Bharti, F. Kloger, C. K. Hall, S. H. L. Klapp, and O. D. Velev, Soft Matter 12, 7747 (2016).

[46] S. Mehdizadeh Taheri, M. Michaelis, T. Friedrich, B. Förster, M. Drechsler, F. M. Römer, P. Bösecke, T. Narayanan, B. Weber, I. Rehberg, S. Rosenfeldt, and S. Förster, Proceedings of the National Academy of Sciences 112, 14484 (2015).

[47] B. V. Derjaguin, Kolloid Zeitschrift 69, 155 (1934).

[48] E. Rabani and S. A. Egorov, The Journal of Chemical Physics 115, 3437 (2001).

[49] E. Rabani and S. A. Egorov, Nano Letters 2, 69 (2002).

[50] U. Landman and W. D. Luedtke, Faraday Discussions 125, 1 (2004).

[51] W. D. Luedtke and U. Landman, The Journal of Physical Chemistry 100, 13323 (1996).

[52] H. N. W. Lekkerkerker and R. Tuinier, Colloids and the depletion interaction, Vol. 833 (Springer, 2011).

[53] K. Park, H. Koerner, and R. A. Vaia, Nano Letters 10, 1433 (2010).

[54] J. Zhang, P. R. Lang, M. Meyer, and J. K. G. Dhont, Langmuir 29, 4679 (2013).

[55] D. Baranov, A. Fiore, M. van Huis, C. Giannini, A. Falqui, U. Lafont, H. Zandbergen, M. Zanella, R. Cingolani, and L. Manna, Nano Letters 10, 743 (2010).

[56] Y. Wang, Y. Wang, X. Zheng, G.-R. Yi, S. Sacanna, D. J. Pine, and M. Weck, Journal of the American Chemical Society 136, 6866 (2014).

[57] M. Gacek, D. Bergsman, E. Michor, and J. C. Berg, Langmuir 28, 11633 (2012).

[58] A. R. Godfrey Alig, M. Akbulut, Y. Golan, and J. Israelachvili, Advanced Functional Materials 16, 2127 (2006).

[59] Y. Min, M. Akbulut, R. K. Prud' homme, Y. Golan, and J. Israelachvili, The Journal of Physical Chemistry B 112, 14395 (2008).

[60] F. Sciortino, G. Casterou, P.-A. Eliat, M.-B. Troadec, C. Gaillard, S. Chevance, M. L. Kahn, and F. Gauffre, ChemNanoMat 2, 759 (2016).

[61] G. A. Jeffrey, An Introduction to Hydrogen Bonding (Oxford University Press, Oxford, 1997).

[62] W. Liu, M. Tagawa, H. L. Xin, T. Wang, H. Emamy, H. Li, K. G. Yager, F. W. Starr, A. V. Tkachenko, and O. Gang, Science 351, 582 (2016).

[63] H. B. G. Casimir and D. Polder, Physical Review 73, 360 (1948).

[64] W. M. R. Simpson, Surprises in Theoretical Casimir Physics, Springer Theses (Springer International Publishing, Cham, 2015).

[65] S. K. Lamoreaux, Physical Review Letters 78, 5 (1997).

[66] G. Bressi, G. Carugno, R. Onofrio, and G. Ruoso, Physical Review Letters 88, 041804 (2002).
[67] B. W. Ninham and J. Daicic, Physical Review A 57, 1870 (1998).

[68] F. S. S. Rosa, D. A. R. Dalvit, and P. W. Milonni, Physical Review A 78, 032117 (2008).

[69] D. Dalvit, P. Milonni, D. Roberts, and F. da Rosa, eds., Casimir Physics, Lecture Notes in Physics, Vol. 834 (Springer Berlin Heidelberg, Berlin, Heidelberg, 2011).

[70] J. N. Munday, F. Capasso, and V. A. Parsegian, Nature 457, 170 (2009).

[71] H. Guo, T. Narayanan, M. Sztuchi, P. Schall, and G. H. Wegdam, Physical Review Letters 100, 188303 (2008).

[72] M. T. Dang, A. V. Verde, V. D. Nguyen, P. G. Bolhuis, and P. Schall, The Journal of Chemical Physics 139, 094903 (2013).

[73] M. E. Fisher and P.-G. de Gennes, Comptes Rendus de l'Academie Des Sciences Serie B 287, 207 (1978).

[74] A. Gambassi, Journal of Physics: Conference Series 161, 012037 (2009).

[75] C. Hertlein, L. Helden, A. Gambassi, S. Dietrich, and C. Bechinger, Nature 451, 172 (2008).

[76] V. D. Nguyen, S. Faber, Z. Hu, G. H. Wegdam, and P. Schall, Nature Communications 4, 2597 (2013).

[77] N. Gnan, E. Zaccarelli, and F. Sciortino, Nature Communications 5, 3267 (2014).

[78] S. Paladugu, A. Callegari, Y. Tuna, L. Barth, S. Dietrich, A. Gambassi, and G. Volpe, Nature Communications 7, 11403 (2016).

[79] I. A. Martínez, C. Devailly, A. Petrosyan, and S. Ciliberto, Entropy 19, 77 (2017).

[80] M. A. Boles, D. Ling, T. Hyeon, and D. V. Talapin, Nature Materials 15, 141 (2016).

[81] M. A. Boles, M. Engel, and D. V. Talapin, Chemical Reviews 116, 11220 (2016).

[82] Z. Huang, P. S. White, and M. Brookhart, Nature 465, 598 (2010).

[83] L.-L. Pontani, M. F. Haase, I. Raczkowska, and J. Brujic, Soft Matter 9, 7150 (2013).

[84] A. B. Pawar and I. Kretzschmar, Macromolecular Rapid Communications 31, 150 (2010).

[85] E. Bianchi, R. Blaak, and C. N. Likos, Physical Chemistry Chemical Physics 13, 6397 (2011).

[86] G.-R. Yi, D. J. Pine, and S. Sacanna, Journal of Physics: Condensed Matter 25, 193101 (2013).

[87] S. Sacanna, D. J. Pine, and G.-R. Yi, Soft Matter 9, 8096 (2013).

[88] E. Duguet, C. Hubert, C. Chomette, A. Perro, and S. Ravaine, Comptes Rendus Chimie 19, 173 (2016).

[89] P. Grammatikopoulos and M. Sowwan, Gas-Phase Synthesis of Nanoparticles (Wiley-VCH Verlag GmbH Co. KGaA, 2017) pp. 269-286.

[90] H. W. Hatch, S.-Y. Yang, J. Mittal, and V. K. Shen, Soft Matter 12, 4170 (2016).

[91] E. Bianchi, J. Largo, P. Tartaglia, E. Zaccarelli, and F. Sciortino, Physical review letters 97, 168301 (2006).

[92] E. Bianchi, P. Tartaglia, E. Zaccarelli, and F. Sciortino, The Journal of Chemical physics 128, 144504 (2008).

[93] Z. Zhang and S. C. Glotzer, Nano Letters 4, 1407 (2004).

[94] Z. Zhang, A. S. Keys, T. Chen, and S. C. Glotzer, Langmuir 21, 11547 (2005).

[95] L. H. Tan, H. Xing, and Y. Lu, Accounts of Chemical Research 47, 1881 (2014).

[96] Y. Kim, R. J. Macfarlane, M. R. Jones, and C. A. Mirkin, Science 351, 579 (2016). 
[97] E. Auyeung, T. I. Li, A. J. Senesi, A. L. Schmucker, B. C. Pals, M. O. de La Cruz, and C. A. Mirkin, Nature 505, 73 (2014).

[98] M. R. Jones, R. J. Macfarlane, B. Lee, J. Zhang, K. L. Young, A. J. Senesi, and C. A. Mirkin, Nature Materials 9, 913 (2010).

[99] S. E. Seo, M. X. Wang, C. M. Shade, J. L. Rouge, K. A. Brown, and C. A. Mirkin, ACS Nano 10, 1771 (2015).

[100] R. R. Mazid, K. J. Si, and W. Cheng, Methods 67, 215 (2014).

[101] C. A. S. Batista, R. G. Larson, and N. A. Kotov, Science 350, 1242477 (2015).

[102] J. H. Conway and N. J. A. Sloane, Sphere packings, lattices and groups, Vol. 290 (Springer Science \& Business Media, 2013).

[103] T. C. Hales, Annals of Mathematics 162, 1065 (2005).

[104] G. van Anders, D. Klotsa, N. K. Ahmed, M. Engel, and S. C. Glotzer, Proceedings of the National Academy of Sciences 111, E4812 (2014).

[105] K. L. Young, M. L. Personick, M. Engel, P. F. Damasceno, S. N. Barnaby, R. Bleher, T. Li, S. C. Glotzer, B. Lee, and C. A. Mirkin, Angewandte Chemie International Edition 52, 13980 (2013).

[106] W. B. Russel, D. A. Saville, and W. R. Schowalter, Colloidal Dispersions (Cambridge University Press, Cambridge, 1989).

[107] M. Elimelech, J. Gregory, X. Jia, and R. A. Williams, Particle Deposition and Aggregation: Measurement, Modeling, and Simulation (Butterworth-Heinemann Ltd., Oxford, 1995).

[108] S. Bhattacharjee, M. Elimelech, and M. Borkovec, Croatica Chemica Acta 71, 883 (1998).

[109] A. V. Tkachenko, Proceedings of the National Academy of Sciences 113, 10269 (2016).

[110] M. J. Murray and J. V. Sanders, Philosophical Magazine A 42, 721 (1980).

[111] M. D. Eldridge, P. A. Madden, and D. Frenkel, Nature 365, 35 (1993).

[112] G. Trefalt and M. Borkovec, http://www.colloid.ch/index.php?name=research (2014).

[113] S. Asakura and F. Oosawa, The Journal of Chemical Physics 22, 1255 (1954).

[114] S. Asakura and F. Oosawa, Journal of Polymer Science 33, 183 (1958).

[115] N. Yang, Z. Yang, M. Held, P. Bonville, P.-A. Albouy, R. Lévy, and M.-P. Pileni, ACS nano 10, 2277 (2016).

[116] R. R. Collino, T. R. Ray, R. C. Fleming, C. H. Sasaki, H. Haj-Hariri, and M. R. Begley, Extreme Mechanics Letters 5, 37 (2015).

[117] P. T. Korda, G. C. Spalding, and D. G. Grier, Physical Review B 66, 024504 (2002).

[118] S.-M. Yang, S.-H. Kim, J.-M. Lim, and G.-R. Yi, Journal of Materials Chemistry 18, 2177 (2008).

[119] M. Volmer and A. Weber, Zeitschrift für Physikalische Chemie (Leipzig) 119, 277 (1926).

[120] R. Becker and W. Döring, Annalen der Physik 416, 719 (1935).

[121] U. Gasser, Journal of Physics: Condensed Matter 21, 203101 (2009).

[122] J. L. Harland and W. Van Megen, Physical Review E 55, 3054 (1997).

[123] K. Schätzel and B. J. Ackerson, Physical Review E 48, 3766 (1993).
[124] S. Auer and D. Frenkel, Nature 409, 1020 (2001).

[125] U. Gasser, E. R. Weeks, A. Schofield, P. N. Pusey, and D. A. Weitz, Science 292, 258 (2001).

[126] B. O' Malley and I. Snook, Physical Review Letters 90, 085702 (2003).

[127] Y. Wang, Y. Wang, X. Zheng, É. Ducrot, J. S. Yodh, M. Weck, and D. J. Pine, Nature Communications 6, 7253 (2015).

[128] W. M. Jacobs and D. Frenkel, Journal of the American Chemical Society 138, 2457 (2016).

[129] A. Reinhardt, C. P. Ho, and D. Frenkel, Faraday Discussions 186, 215 (2016).

[130] H. Yan, S. Cingarapu, K. J. Klabunde, A. Chakrabarti, and C. M. Sorensen, Physical Review Letters 102, 095501 (2009).

[131] S. G. Kwon and T. Hyeon, Small 7, 2685 (2011).

[132] V. K. LaMer and R. H. Dinegar, Journal of the American Chemical Society 72, 4847 (1950).

[133] G. M. Lothe and J. Pound, The Journal of Chemical Physics 36, 2080 (1962).

[134] N. T. K. Thanh, N. Maclean, and S. Mahiddine, Chemical Reviews 114, 7610 (2014).

[135] R. Viswanatha and D. D. Sarma, in Nanomaterials Chemistry, edited by C. N. R. Rao, A. Müller, and A. K. Cheetham (Wiley-VCH Verlag GmbH \& Co. KGaA, 2007) pp. 139-170.

[136] A. Baldan, Journal of Materials Science 37, 2171 (2002).

[137] V. Radmilovic, C. Ophus, E. A. Marquis, M. D. Rossell, A. Tolley, A. Gautam, M. Asta, and U. Dahmen, Nature Materials 10, 710 (2011).

[138] C. J. Kuehmann and P. W. Voorhees, Metallurgical and Materials Transactions A 27, 937 (1996).

[139] W.-r. Lee, M. G. Kim, J.-r. Choi, J.-I. Park, S. J. Ko, S. J. Oh, and J. Cheon, Journal of the American Chemical Society 127, 16090 (2005).

[140] Y. Xia, T. D. Nguyen, M. Yang, B. Lee, A. Santos, P. Podsiadlo, Z. Tang, S. C. Glotzer, and N. A. Kotov, Nature nanotechnology 6, 580 (2011).

[141] J. E. Funk and D. Dinger, Predictive process control of crowded particulate suspensions: applied to ceramic manufacturing (Springer Science \& Business Media, 2013).

[142] E. Dickinson and R. Parker, Journal de Physique Lettres 46, 229 (1985).

[143] P. N. Pusey, Journal de Physique 48, 709 (1987).

[144] M. Fasolo and P. Sollich, Physical Review E 70, 041410 (2004).

[145] M. Fasolo and P. Sollich, Physical Review Letters 91, 068301 (2003).

[146] M. D. Rintoul and S. Torquato, The Journal of Chemical Physics 105, 9258 (1996).

[147] M. Gardner, The colossal book of mathematics: classic puzzles, paradoxes, and problems: number theory, algebra, geometry, probability, topology, game theory, infinity, and other topics of recreational mathematics (WW Norton \& Company, 2001).

[148] C. Song, P. Wang, and H. A. Makse, Nature 453, 629 (2008).

[149] V. N. Manoharan and D. J. Pine, MRS Bulletin 29, 91 (2004).

[150] U. Gasser, Journal of Physics: Condensed Matter 21, 203101 (2009). 
[151] V. J. Anderson and H. N. Lekkerkerker, Nature 416, 811 (2002).

[152] J. Largo, M. A. Miller, and F. Sciortino, The Journal of Chemical Physics 128, 134513 (2008).

[153] M. A. Miller and D. Frenkel, Physical Review Letters 90, 135702 (2003).

[154] M. Gardner, New mathematical diversions (Mathematical Association of America, 1995).

[155] M. A. Boles and D. V. Talapin, Journal of the American Chemical Society 136, 5868 (2014).

[156] P. Chaikin, S. Wang, and A. Jaoshvili, in APS Meeting Abstracts (2007).

[157] E. R. Chen, Discrete \& Computational Geometry 40, 214 (2008).

[158] S. Torquato and Y. Jiao, Physical Review E 80, 041104 (2009).

[159] E. R. Chen, M. Engel, and S. C. Glotzer, Discrete \& Computational Geometry 44, 253 (2010).

[160] S. Torquato and Y. Jiao, Physical Review E 81, 041310 (2010).

[161] P. F. Damasceno, M. Engel, and S. C. Glotzer, Science 337, 453 (2012).

[162] S. Torquato and Y. Jiao, Physical Review E 86, 011102 (2012).

[163] J. Henzie, M. Grünwald, A. Widmer-Cooper, P. L. Geissler, and P. Yang, Nature Materials 11, 131 (2012).

[164] A. Haji-Akbari, M. Engel, A. S. Keys, X. Zheng, R. G. Petschek, P. Palffy-Muhoray, and S. C. Glotzer, Nature 462, 773 (2009).

[165] Y. Kallus, V. Elser, and S. Gravel, Discrete \& Computational Geometry 44, 245 (2010).

[166] R. Gabbrielli, Y. Jiao, and S. Torquato, Physical Review E 86, 041141 (2012).

[167] J. de Graaf, R. van Roij, and M. Dijkstra, Physical Review Letters 107, 155501 (2011).

[168] A. Donev, F. H. Stillinger, P. M. Chaikin, and S. Torquato, Physical Review Letters 92, 255506 (2004).

[169] A. Kuijk, D. V. Byelov, A. V. Petukhov, A. v. Blaaderen, and A. Imhof, Faraday Discussions 159, 181 (2013).

[170] A. P. Gantapara, J. de Graaf, R. van Roij, and M. Dijkstra, Physical Review Letters 111, 015501 (2013).

[171] B. De Nijs, S. Dussi, F. Smallenburg, J. D. Meeldijk, D. J. Groenendijk, L. Filion, A. Imhof, A. Van Blaaderen, and M. Dijkstra, Nature Materials 14, 56 (2015).

[172] A. F. de Moura, K. Bernardino, C. J. Dalmaschio, E. R. Leite, and N. A. Kotov, Physical Chemistry Chemical Physics 17, 3820 (2015).

[173] A. Widmer-Cooper and P. L. Geissler, ACS Nano 10, 1877 (2016).

[174] J. Zhang, Z. Luo, Z. Quan, Y. Wang, A. Kumbhar, D.M. Smilgies, and J. Fang, Nano Letters 11, 2912 (2011).

[175] R. Li, J. Zhang, R. Tan, F. Gerdes, Z. Luo, H. Xu, J. A. Hollingsworth, C. Klinke, O. Chen, and Z. Wang, Nano Letters 16, 2792 (2016).

[176] J. Zhang, Z. Luo, B. Martens, Z. Quan, A. Kumbhar, N. Porter, Y. Wang, D.-M. Smilgies, and J. Fang, Journal of the American Chemical Society 134, 14043 (2012).

[177] S. Xie, X. Zhou, X. Han, Q. Kuang, M. Jin, Y. Jiang, Z. Xie, and L. Zheng, The Journal of Physical Chemistry C 113, 19107 (2009).
[178] T. R. Gordon, T. Paik, D. R. Klein, G. V. Naik, H. Caglayan, A. Boltasseva, and C. B. Murray, Nano Letters 13, 2857 (2013).

[179] X. S. Shen, G. Z. Wang, X. Hong, and W. Zhu, CrystEngComm 11, 753 (2009).

[180] Y. Zhang, F. Lu, D. van der Lelie, and O. Gang, Physical Review Letters 107, 135701 (2011).

[181] H. Chan, A. Demortière, L. Vukovic, P. Král, and C. Petit, ACS Nano 6, 4203 (2012).

[182] Z. Quan, H. Xu, C. Wang, X. Wen, Y. Wang, J. Zhu, R. Li, C. J. Sheehan, Z. Wang, D.-M. Smilgies, Z. Luo, and J. Fang, Journal of the American Chemical Society 136, 1352 (2014).

[183] J. J. Choi, K. Bian, W. J. Baumgardner, D.-M. Smilgies, and T. Hanrath, Nano Letters 12, 4791 (2012).

[184] T. Wang, J. Zhuang, J. Lynch, O. Chen, Z. Wang, X. Wang, D. LaMontagne, H. Wu, Z. Wang, and Y. C. Cao, Science 338, 358 (2012).

[185] S. Disch, E. Wetterskog, R. P. Hermann, G. SalazarAlvarez, P. Busch, T. Bruckel, L. Bergstrom, and S. Kamali, Nano Letters 11, 1651 (2011).

[186] H. J. H. Brouwers, Physical Review E 76, 041304 (2007).

[187] H. J. H. Brouwers, Physical Review E 78, 011303 (2008).

[188] A.-P. Hynninen, J. H. J. Thijssen, E. C. M. Vermolen, M. Dijkstra, and A. van Blaaderen, Nature Materials 6, 202 (2007).

[189] F. Lu, K. G. Yager, Y. Zhang, H. Xin, and O. Gang, Nature Communications 6, 6912 (2015).

[190] É. Ducrot, M. He, G.-R. Yi, and D. J. Pine, Nature Materials (2017).

[191] C. Yan and T. Wang, Chemical Society Reviews 46, 1483 (2017).

[192] F. García-Santamaría, H. Miyazaki, A. Urquía, M. Ibisate, M. Belmonte, N. Shinya, F. Meseguer, and C. López, Advanced Materials 14, 1144 (2002).

[193] R. Hieronimus, S. Raschke, and A. Heuer, The Journal of Chemical Physics 145, 064303 (2016).

[194] L. Hong, A. Cacciuto, E. Luijten, and S. Granick, Langmuir 24, 621 (2008).

[195] N. Kern and D. Frenkel, The Journal of Chemical Physics 118, 9882 (2003).

[196] F. Sciortino, E. Bianchi, J. F. Douglas, and P. Tartaglia, The Journal of Chemical Physics 126, 194903 (2007).

[197] M. S. Wertheim, Journal of Statistical Physics 42, 459 (1986).

[198] Z. Zhang and S. C. Glotzer, Nano Letters 4, 1407 (2004).

[199] F. Romano and F. Sciortino, Soft Matter 7, 5799 (2011).

[200] D. Z. Rocklin and X. Mao, Soft Matter 10, 7569 (2014).

[201] F. Romano and F. Sciortino, Nature Communications 3, 975 (2012).

[202] A. B. Pawar and I. Kretzschmar, Langmuir 25, 9057 (2009).

[203] D. J. Ashton, R. L. Jack, and N. B. Wilding, Soft Matter 9, 9661 (2013).

[204] F. Romano, P. Tartaglia, and F. Sciortino, Journal of Physics: Condensed Matter 19, 322101 (2007).

[205] F. Romano, E. Sanz, and F. Sciortino, The Journal of Chemical Physics 132, 184501 (2010).

[206] G. Doppelbauer, E. G. Noya, E. Bianchi, and G. Kahl, Journal of Physics: Condensed Matter 24, 284124 
(2012).

[207] E. Bianchi, G. Doppelbauer, L. Filion, M. Dijkstra, and G. Kahl, The Journal of Chemical Physics 136, 214102 (2012).

[208] F. Smallenburg and F. Sciortino, Nature Physics 9, 554 (2013).

[209] G. van Anders, N. K. Ahmed, R. Smith, M. Engel, and S. C. Glotzer, ACS Nano 8, 931 (2014).

[210] J. Chang, A. M. Lenhoff, and S. I. Sandler, The Journal of Chemical Physics 120, 3003 (2004).

[211] J. P. K. Doye, A. A. Louis, I.-C. Lin, L. R. Allen, E. G. Noya, A. W. Wilber, H. C. Kok, and R. Lyus, Physical Chemistry Chemical Physics 9, 2197 (2007).

[212] E. G. Noya, C. Vega, J. P. K. Doye, and A. A. Louis, The Journal of Chemical Physics 127, 054501 (2007).

[213] Z. Nie, D. Fava, E. Kumacheva, S. Zou, G. C. Walker, and M. Rubinstein, Nature Materials 6, 609 (2007).

[214] S. Sacanna, W. T. M. Irvine, P. M. Chaikin, and D. J. Pine, Nature 464, 575 (2010).

[215] Q. Chen, S. C. Bae, and S. Granick, Nature 469, 381 (2011).

[216] S.-H. Kim, G.-R. Yi, K. H. Kim, and S.-M. Yang, Langmuir 24, 2365 (2008)

[217] D. J. Kraft, W. S. Vlug, C. M. van Kats, A. van Blaaderen, A. Imhof, and W. K. Kegel, Journal of the American Chemical Society 131, 1182 (2009).

[218] V. Meester, R. W. Verweij, C. van der Wel, and D. J. Kraft, ACS Nano 10, 4322 (2016).

[219] E. Lauga and M. P. Brenner, Physical Review Letters 93, 238301 (2004).

[220] V. N. Manoharan, Science 301, 483 (2003).

[221] G.-R. Yi, V. N. Manoharan, E. Michel, M. T. Elsesser, S.-M. Yang, and D. J. Pine, Advanced Materials 16, 1204 (2004).

[222] Y.-S. Cho, G.-R. Yi, S.-H. Kim, S.-J. Jeon, M. T. Elsesser, H. K. Yu, S.-M. Yang, and D. J. Pine, Chemistry of Materials 19, 3183 (2007).

[223] Y. Wang, Y. Wang, D. R. Breed, V. N. Manoharan, L. Feng, A. D. Hollingsworth, M. Weck, and D. J. Pine, Nature 491, 51 (2012).

[224] A. Perro, E. Duguet, O. Lambert, J.-C. Taveau, E. Bourgeat-Lami, and S. Ravaine, Angewandte Chemie International Edition 48, 361 (2009).

[225] A. Désert, I. Chaduc, S. Fouilloux, J.-C. Taveau, O. Lambert, M. Lansalot, E. Bourgeat-Lami, A. Thill, O. Spalla, S. Ravaine, and E. Duguet, Polymer Chemistry 3, 1130 (2012).

[226] A. Désert, C. Hubert, Z. Fu, L. Moulet, J. Majimel, P. Barboteau, A. Thill, M. Lansalot, E. Bourgeat-Lami, E. Duguet, and S. Ravaine, Angewandte Chemie International Edition 52, 11068 (2013).

[227] C. Hubert, C. Chomette, A. Désert, M. Sun, M. Treguer-Delapierre, S. Mornet, A. Perro, E. Duguet, and S. Ravaine, Faraday Discuss. 181, 139 (2015).

[228] A. Désert, J. Morele, J.-C. Taveau, O. Lambert, M. Lansalot, E. Bourgeat-Lami, A. Thill, O. Spalla, L. Belloni, S. Ravaine, and E. Duguet, Nanoscale 8, 5454 (2016).

[229] C. Chomette, P. E. Rouet, L. Adumeau, M. TreguerDelapierre, S. Mornet, S. Ravaine, and E. . Duguet, "unpublished results,".

[230] S. Y. Park, A. K. R. Lytton-Jean, B. Lee, S. Weigand, G. C. Schatz, and C. A. Mirkin, Nature 451, 553 (2008).
[231] D. Nykypanchuk, M. M. Maye, D. Van Der Lelie, and O. Gang, Nature 451, 549 (2008).

[232] E. Auyeung, J. I. Cutler, R. J. Macfarlane, M. R. Jones, J. Wu, G. Liu, K. Zhang, K. D. Osberg, and C. A. Mirkin, Nature Nanotechnology 7, 24 (2012).

[233] M. B. Zanjani, I. C. Jenkins, J. C. Crocker, and T. Sinno, ACS Nano 10, 11280 (2016).

[234] J. D. Halverson and A. V. Tkachenko, Physical Review E 87, 062310 (2013).

[235] M. N. O' Brien, H.-X. Lin, M. Girard, M. Olvera de la Cruz, and C. A. Mirkin, Journal of the American Chemical Society 138, 14562 (2016).

[236] D. Frenkel, Nature Materials 14, 9 (2015).

[237] W. M. Jacobs, A. Reinhardt, and D. Frenkel, Proceedings of the National Academy of Sciences 112, 6313 (2015).

[238] W. M. Jacobs and D. Frenkel, Soft Matter 11, 8930 (2015).

[239] A. Kuzyk, R. Schreiber, Z. Fan, G. Pardatscher, E.-M. Roller, A. Högele, F. C. Simmel, A. O. Govorov, and T. Liedl, Nature 483, 311 (2012).

[240] T. Tigges, T. Heuser, R. Tiwari, and A. Walther, Nano Letters 16, 7870 (2016).

[241] S. Pal, R. Varghese, Z. Deng, Z. Zhao, A. Kumar, H. Yan, and Y. Liu, Angewandte Chemie International Edition 50, 4176 (2011).

[242] H. Y. Chen, S. Abraham, J. Mendenhall, S. C. Delamarre, K. Smith, I. Kim, and C. A. Batt, ChemPhysChem 9, 388 (2008).

[243] L. H. Tan, H. Xing, H. Chen, and Y. Lu, Journal of the American Chemical Society 135, 17675 (2013).

[244] S. C. Glotzer and M. J. Solomon, Nature Materials 6, 557 (2007).

[245] O. Gang, Faraday Discussions 186, 529 (2016).

[246] S. John, Physics Today 44, 32 (1991).

[247] E. Yablonovitch, Journal of the Optical Society of America B 10, 283 (1993).

[248] J. D. Joannopoulos, S. G. Johnson, J. N. Winn, and R. D. Meade, Photonic crystals: molding the flow of light (Princeton university press, 2011).

[249] J. D. Joannopoulos, P. R. Villeneuve, and S. Fan, Nature 386, 143 (1997)

[250] A. Scherer, T. Doll, E. Yablonovitch, H. O. Everitt, and J. A. Higgins, Journal of Lightwave Technology 17, 1928 (1999).

[251] H. Cong, B. Yu, J. Tang, Z. Li, and X. Liu, Chemical Society Reviews 42, 7774 (2013).

[252] G. von Freymann, V. Kitaev, B. V. Lotsch, and G. A. Ozin, Chemical Society Reviews 42, 2528 (2013).

[253] H. Zheng and S. Ravaine, Crystals 6, 54 (2016).

[254] E. Yablonovitch, Physical Review Letters 58, 2059 (1987).

[255] S. John, Physical Review Letters 58, 2486 (1987).

[256] K. M. Leung and Y. F. Liu, Physical Review Letters 65, 2646 (1990).

[257] Z. Zhang and S. Satpathy, Physical Review Letters 65, 2650 (1990)

[258] H. S. Sözüer, J. W. Haus, and R. Inguva, Physical Review B 45, 13962 (1992).

[259] J. W. Haus, Journal of Modern Optics 41, 195 (1994).

[260] V. Lousse, J.-P. Vigneron, X. Bouju, and J.-M. Vigoureux, Physical Review B 64, 201104(R) (2001).

[261] Z.-Y. Li, J. Wang, and B.-Y. Gu, Journal of the Physical Society of Japan 67, 3288 (1998). 
[262] Y. Xia, B. Gates, and Z.-Y. Li, Advanced Materials 13, 409 (2001).

[263] Z.-Y. Li, J. Wang, and B.-Y. Gu, Physical Review B 58, 3721 (1998).

[264] S. Simeonov, U. Bass, and A. R. McGurn, Physica B: Condensed Matter 228, 245 (1996).

[265] I. D. Hosein, M. Ghebrebrhan, J. D. Joannopoulos, and C. M. Liddell, Langmuir 26, 2151 (2010).

[266] J.-W. Kim, R. J. Larsen, and D. A. Weitz, Advanced Materials 19, 2005 (2007).

[267] S. Reculusa, C. Poncet-Legrand, A. Perro, E. Duguet, E. Bourgeat-Lami, C. Mingotaud, and S. Ravaine, Chemistry of Materials 17, 3338 (2005).

[268] I. D. Hosein, S. H. Lee, and C. M. Liddell, Advanced Functional Materials 20, 3085 (2010).

[269] K. Ho, T. Chan, and C. Soukoulis, Physical Review Letters 65, 3152 (1990).

[270] M. Maldovan, C. K. Ullal, W. C. Carter, and E. L. Thomas, Nature Materials 2, 664 (2003).

[271] A. Moroz, Physical Review B 66, 115109 (2002).

[272] A. Garcia-Adeva, New Journal of Physics 8, 86 (2006).

[273] Z.-Y. Li and Z.-Q. Zhang, Physical Review B 62, 1516 (2000).

[274] T. T. Ngo, C. M. Liddell, M. Ghebrebrhan, and J. D. Joannopoulos, Applied Physics Letters 88, 241920 (2006).

[275] E. C. Nelson and P. V. Braun, Science 318, 924 (2007).

[276] D. R. Nelson, Nano Letters 2, 1125 (2002).

[277] X. Mao, Q. Chen, and S. Granick, Nature Materials 12, 217 (2013).

[278] D. R. Smith, W. J. Padilla, D. Vier, S. C. Nemat-Nasser, and S. Schultz, Physical Review Letters 84, 4184 (2000).

[279] J. Hunt, T. Driscoll, A. Mrozack, G. Lipworth, M. Reynolds, D. Brady, and D. R. Smith, Science 339, 310 (2013).

[280] A. Tao, P. Sinsermsuksakul, and P. Yang, Nature Nanotechnology 2, 435 (2007).

[281] C.-F. Chen, S.-D. Tzeng, H.-Y. Chen, K.-J. Lin, and S. Gwo, Journal of the American Chemical Society 130, 824 (2008).

[282] J. Chao, Y. Lin, H. Liu, L. Wang, and C. Fan, Materials Today 18, 326 (2015).

[283] M. B. Ross, J. C. Ku, V. M. Vaccarezza, G. C. Schatz, and C. A. Mirkin, Nature nanotechnology 10, 453 (2015).

[284] Y. Wang, H. Arandiyan, J. Scott, A. Bagheri, H. Dai, and R. Amal, Journal of Materials Chemistry A 5, 8825 (2017).

[285] C. R. Henry, Catalysis Letters 145, 731 (2015).

[286] K. R. Phillips, G. T. England, S. Sunny, E. Shirman, T. Shirman, N. Vogel, and J. Aizenberg, Chemical Society Reviews 45, 281 (2016).

[287] H. Arandiyan, H. Dai, K. Ji, H. Sun, and J. Li, ACS Catalysis 5, 1781 (2015).

[288] Y. Wang, H. Arandiyan, J. Scott, M. Akia, H. Dai, J. Deng, K.-F. Aguey-Zinsou, and R. Amal, ACS Catalysis 6, 6935 (2016), http://dx.doi.org/10.1021/acscatal.6b01685.

[289] Y. Wei, J. Jiao, X. Zhang, B. Jin, Z. Zhao, J. Xiong, Y. Li, J. Liu, and J. Li, Nanoscale 9, 4558 (2017).

[290] J. Jiao, Y. Wei, Z. Zhao, W. Zhong, J. Liu, J. Li, A. Duan, and G. Jiang, Catalysis Today 258, 319
(2015).

[291] C.-T. Dinh, H. Yen, F. Kleitz, and T.-O. Do, Angewandte Chemie International Edition 53, 6618 (2014).

[292] E. Morsbach, S. Kunz, and M. Bäumer, Catalysis Science \& Technology 6, 339 (2016).

[293] M. Curti, J. Schneider, D. W. Bahnemann, and C. B. Mendive, The journal of physical chemistry letters $\mathbf{6}$, 3903 (2015).

[294] M.-P. Pileni, Journal of Physics D: Applied Physics 41, 134002 (2008).

[295] E. C. Stoner and E. P. Wohlfarth, Philosophical Transactions of the Royal Society A: Physical, Mathematical and Engineering Sciences 240, 599 (1948).

[296] S. A. Claridge, A. W. Castleman Jr, S. N. Khanna, C. B. Murray, A. Sen, and P. S. Weiss, Acs Nano 3, 244 (2009).

[297] S. A. Majetich, T. Wen, and R. A. Booth, ACS Nano 5, 6081 (2011).

[298] G. Reiss and A. Hütten, Nature Materials 4, 725 (2005).

[299] H. Zeng, J. Li, J. P. Liu, Z. L. Wang, and S. Sun, Nature 420, 395 (2002).

[300] M.-P. Pileni, Surface Science 603, 1498 (2009).

[301] M.-P. Pileni, Journal of Materials Chemistry 21, 16748 (2011).

[302] J. Ge, Y. Hu, and Y. Yin, Angewandte Chemie International Edition 46, 7428 (2007).

[303] X. Guo, B. Sun, J. Zhang, H. Liu, and G. Wang, Journal of Materials Chemistry A 4, 9774 (2016).

[304] M. El Ghezal, Y. Maalej, and I. Doghri, Computational Materials Science 70, 51 (2013).

[305] T. J. Willmore, Riemannian geometry (1996).

[306] A. I. Bobenko, Combinatorial and computational geometry 52, 133 (2005).

[307] W. Helfrich, Zeitschrift für Naturforschung C 28, 693 (1973).

[308] Y. Wei, B. Wang, J. Wu, R. Yang, and M. L. Dunn, Nano letters 13, 26 (2012).

[309] L. Yu and C. Ru, Mathematics and Mechanics of Solids , 1081286515597735 (2015).

[310] D. W. Abueidda, R. K. A. Al-Rub, A. S. Dalaq, D.-W. Lee, K. A. Khan, and I. Jasiuk, Mechanics of Materials 95, 102 (2016).

[311] R. Bruinsma, Journal de Physique II 2, 425 (1992).

[312] D. C. Miller, M. Terrones, and H. Terrones, Carbon 96, 1191 (2016).

[313] Z. Qin, G. S. Jung, M. J. Kang, and M. J. Buehler, Science Advances 3 (2017), 10.1126/sciadv.1601536, http://advances.sciencemag.org/content/3/1/e1601536.full.pdf.

[314] I. Gibson, D. Rosen, and B. Stucker, Additive manufacturing technologies: 3D printing, rapid prototyping, and direct digital manufacturing (Springer, 2014).

[315] J.-M. Lehn, Angewandte Chemie International Edition 52, 2836 (2013).

[316] L. Cademartiri and K. J. Bishop, Nature Materials 14, 2 (2015).

[317] W. B. Rogers, W. M. Shih, and V. N. Manoharan, Nature Reviews Materials 1, 16008 (2016).

[318] Z. Zeravcic, V. N. Manoharan, and M. P. Brenner, Proceedings of the National Academy of Sciences 111, 15918 (2014). 